T. HASEGAWA

KODAI MATH. J.

3 (1.980), 224-252

\title{
SPECTRAL GEOMETRY OF CLOSED MINIMAL SUBMANIFOLDS IN A SPACE FORM, REAL OR COMPLEX
}

\author{
By TAKUICHI HASEGAWA
}

\section{$\S 0$. Introduction.}

Let $N$ be a Riemannian space, $M$ be a minimal submanifold of $N$, and $D$ be a compact domain of $M$. Thinking of $D$ as an equilibrium state of a homogeneous membrane with its boundary fixed, and expressing a small motion of $D$ by normal vector fields through the normal exponential mapping, we derived in [11] the equation of a vibrating general membrane $D$. By the separation of variables, we obtain a generalization of the Helmholtz equation, $J V=\lambda V$ on $D$, where $V$ is a normal vector field on $D$ vanishing on the boundary and $J$ is the Jacobi differential operator. Then we call the complete set of eigenvalues of $J$ simply the spectrum of the minimal submanifold $D$. Thus there arises eigenvalue problem of compact minimal submanifolds. In this paper we shall study the inverse eigenvalue problem (i. e. spectral geometry), when the ambient space $N$ is a space of constant curvature or of constant holomorphic curvature. Every minimal submanifold of $N$ we consider in this paper is assumed to be without boundary. Some studies along this line have already been done by $\mathrm{H}$. Donnelly [8], J. Simons [24] and others.

In $\S 1$ we make preliminaries. We give there definitions and notations, some lemmas and some examples of compact minimal submanifolds. In $\S 2$ we estimate the first eigenvalue of $J$ by means of geometric quantities. In $\S 3$ we review a Gikey's paper. In $\S 4$, making use of his results, we clarify the geometric meaning of the first three terms of the asymptotic expansion for $t \downarrow 0$ of the partition function $\Sigma e^{-\lambda_{i} t}$, and then obtain Riemannian and Kaehlerian spectral invariants. In $\S 5$, using spectral invariants given in $\S 4$, we obtain some properties which are derived from or reflected under the isospectral condition. Then by these isospectral properties we characterize some concrete minimal submanifolds in a sphere, particularly Veronese manifolds. In $\S 6$ we study the Kaehlerian version of $\S 5$.

The author would like to express his hearty gratitude to Professor S. Ishihara, Professor T. Otsuki and Dr. K. Sakamoto for their encouragements and valuable suggestions.

Received April 14, 1979 


\section{$\S 1$. Preliminaries.}

Throughout this paper except in $\S 6$ and unless otherwise stated, $N=N(c)$ will denote an $n$-dimensional Riemannian manifold of constant section curvature c. All minimal submanifolds of $N$, which appear in this paper, are assumed to have no boundary and to be compact, connected, and of class $C^{\infty}$. (We note that some of definitions and notions stated below are still valid for an arbitrary Riemannian manifold $N$ and its arbitrary submanifold $M$ ).

Let $M$ be such an $m$-dimensional minimal submanifold of $N$ and let $g, R, \rho$ and $\tau$ be respectively the metric tensor, curvature tensor, Ricci tensor and scalar curvature of $M$. We denote by $R_{\imath \jmath k l}$ and so on the components of $R$ and so on with respect to a natural frame of a tangent space $T_{p} M$. (i.e. $R_{\imath j k l}=$ $\left.\left\langle\left(\nabla_{j} \nabla_{i}-\nabla_{i} \nabla_{j}\right) \partial / \partial x_{k}, \partial / \partial x_{l}\right\rangle\right)$. Let $C$ be the Weyl's conformal curvature tensor of $M$, which is given by

$$
\begin{aligned}
C_{\imath j k l}= & R_{\imath j k l}-\frac{1}{m-2}\left(\rho_{j k} g_{i l}-\rho_{\jmath l} g_{\imath k}+g_{j k} \rho_{\imath l}-g_{\jmath l} \rho_{\imath k}\right) \\
& +\frac{1}{(m-1)(m-2)}\left(g_{\jmath k} g_{\imath l}-g_{j l} g_{\imath k}\right) \tau,
\end{aligned}
$$

and let $G$ be a 2-covariant tensor such that

$$
G=\rho-\frac{\tau}{m} g .
$$

In general we denote by || the norm of a tensor with respect to the appropriate inner product $\langle$,$\rangle . Then$

$$
\begin{gathered}
|C|^{2}=|R|^{2}-\frac{4}{m-2}|\rho|^{2}+\frac{2}{(m-1)(m-2)} \tau^{2}, \\
|G|^{2}=|\rho|^{2}-\frac{1}{m} \tau^{2} .
\end{gathered}
$$

Thus $G=0$ holds if and only if $M$ is Einstein. And thus $C=0$ and $G=0$ hold if and only if $M$ has a constant sectional curvature $(m \geqq 4)$.

Let $\bar{\nabla}$ and $\nabla$ be the canonical covariant derivations in $N$ and $M$, respectively. Let $T M^{\perp}$ be the normal bundle of $M$ in $N . T M^{\perp}$ is a Riemannian vector bundle and its canonical covariant derivation (i. e. the normal connection) is also denoted by $\nabla$. These are related as follows:

$$
\bar{\nabla}_{X} Y=\nabla_{X} Y+B(X, Y), \quad \bar{\nabla}_{X} V=\nabla_{X} V-A^{V}(X), \quad\langle V, B(X, Y)\rangle=\left\langle A^{V}(X), Y\right\rangle
$$

for tangent vector fields $X, Y$ on $M$ and a normal vector field $V$. The tensor field $A$ is called the second fundamental tensor of $M$, and is a cross section of the Riemannian vector bundle $\operatorname{Hom}\left(T M^{\perp}, S M\right)$, where $S M$ is the bundle of symmetric transformations of the tangent bundle $T M$. The composition of $A$ 
and its transpose ${ }^{t} A$ is denoted by $\tilde{A}$, that is, $\tilde{A}={ }^{t} A \circ A \in C^{\infty}\left(\operatorname{Hom}\left(T M^{\perp}, T M^{\perp}\right)\right)$. The trace of $\tilde{A}$, i. e. square norm $|A|^{2}$ of the second fundamental tensor $A$ is denoted by $S$. The trace of $\tilde{A} \circ \tilde{A}$, i. e. square norm $|\tilde{A}|^{2}$ of $\tilde{A}$ is denoted by $L_{N}$. When $M$ is of codimension $1, L_{N}=S^{2}$ holds (in a general case $L_{N} \leqq S^{2}$ holds, and the equality is attained if and only if $m$-index of $M$ is equal to 0 or 1 at each point of $M$ ). We denote by $K_{N}$ the square norm of the curvature tensor of the normal connections, which is called the normal scalar curvature of the immersion. $S, L_{N}$ and $K_{N}$ are nonnegative $C^{\infty}$ functions on $M$. Let $\bar{R}$ be the curvature tensor of $N$. And let $\widetilde{R}$ be a sort of partial Ricci transformation, which is defined by $\tilde{R}(v):=\sum_{\imath=1}^{m}\left(\bar{R}_{e_{1}, v} e_{\imath}\right)^{\perp}$, where $v$ is a normal vector at $p$, $\left(e_{1}, \cdots, e_{m}\right)$ an orthonormal frame of $T M_{p}$ and ()$^{\perp}$ denotes the normal part of a vector. In case $N$ is a space of constant curvature $c, \widetilde{R}$ is a scalar transformation: $\tilde{R}=-m c I$. In general we denote by $\nabla^{2}$ the laplace operator (which is also called the restricted Laplacian) acting on cross sections of a Riemannian vector bundle. Let us consider the differential operator $J$ defined by

$$
J=-\nabla^{2}+\tilde{R}-\tilde{A}
$$

which acts on normal vector field of $M$. In this paper we call this operator the Jacobi differential operator. The $J$ arose from the second variation formula of $M$, namely, for a normal vector field $V$ on $M, \int_{M}\langle J V, V\rangle$ gives just the second variation of $M$ with respect to the variation vector field $V$ ([24]). $J$ is selfadjoint, strongly elliptic of second order and has a discrete spectrum. We call the complete set of eigenvalues of $J$ simply the spectrum of the minimal submanifold $M$ and denote it by $\operatorname{Spec}(M, N)=\left\{\lambda_{1} \leqq \lambda_{2} \leqq \lambda_{3} \leqq \cdots \rightarrow \infty\right\}$. A geometric quantity is called a "spectral invariant" when it is determined by the spectrum, and a geometric property is called a "spectral property" when it is reflected under the isospectral condition. Then the fundamental problem of the inverse eigenvalue problem is how far the spectrum determines geometric properties of the minimal submanifold. The analogous problem for the case of the LaplaceBeltrami operator of compact Riemannian manifolds has been variously studied by many authors.

We denote by $T$ the square norm of the covariant derivative of the second fundamental tensor $A ; T=|\nabla A|^{2}$.

If $M^{\prime}$ denotes another minimal submanifold of $N$, then $R^{\prime}, \rho^{\prime}, \tau^{\prime}, K_{N}^{\prime}$ and so on denote the corresponding quantities of $M^{\prime}$.

An isometric immersion is said to be full if it is not contained in any totally geodesic submanifold. For a unit tangent vector $x, B(x, x)$ is called the normal curvature vector determined by $x$. An immersion is said to be $\imath$ sotropic if at each point every normal curvature vector has the same length.

Let $p$ be a point of $M$. Then m-index at $p$ of a minimal submanifold $M$ is defined by the rank of the second fundamental tensor $A$ at $p$ as a linear mapping from $T M_{p}^{\perp}$ to $S M_{p}$.

If $f$ is a function on $M$, the integral $\int_{M} f$ of $f$ over $M$ is also denoted by 
$f(M)$, where the integration is of course carried out with respect to the canonical measure of the Riemannian manifold $M$.

Now we exhibit some examples of minimal submanifolds in a sphere and state their characteristic properties. Let $S^{m}(r)$ be the $m$-dimensional sphere of radius $r$ in $R^{m+1}$. $S^{m}(1)$ is simply written as $S^{m}$. Any totally geodesic submanifold of $S^{n}$ is again a sphere of radius 1 .

Let $p$ be a positive integer $(1 \leqq p \leqq[m / 2])$. By considering $S^{p}(\sqrt{ } p / m) \times$ $S^{m-p}(\sqrt{ } m-p / p)$ as a natural subspace of $R^{p+1} \times R^{m-p+1}=R^{m+2}$, we obtain a natural map from $S^{p}(\sqrt{p / m}) \times S^{m-p}(\sqrt{m-p / m})$ to $S^{m+1}$. We denote by $M_{p, m-p}$ the manifold $S^{p}(\sqrt{p / m}) \times S^{m-p}(\sqrt{m-p / m})$ together with this mapping. They are called $m$-dimensional Clifford hypersurfaces of $S^{m+1}$ (or generalized Clifford torus). In particular $M_{1,1}$ is called the Clifford torus, which is flat. It is easy to see that they are minimal submanifolds of $S^{m+1}$ with parallel second fundamental tensor and $S=m$. There are a number of properties which characterize this class $M_{p, m-p}$. One of them is the following; a compact minimal hypersurface of $S^{m+1}$ with $S=m$ is an $m$-dimensional Clifford hypersurface ([5], [19]).

Clifford hypersurfaces are generalized as follows. Let $m_{1}, \cdots, m_{k}$ be positive integers and $m=m_{1}+\cdots+m_{k}$. Let $x_{\imath}$ be a point of $S^{m_{i}}\left(\sqrt{m_{\imath} / m}\right)$ i. e. a vector of length $\sqrt{m_{\imath} / m}$ in $R^{m_{\imath}+1}$. Then $\left(x_{1}, \cdots, x_{k}\right)$ is a unit vector in $R^{m+k}$. This defines a mapping from $S^{m_{1}}\left(\sqrt{m_{1} / m}\right) \times \cdots \times S^{m_{k}}\left(\sqrt{m_{k} / m}\right)$ to $S^{m+k-1}$, which is denoted by $M_{m_{1}, \ldots, m_{k}}$. It is easy to see that it is a minimal submanifold with parallel second fundamental tensor, its normal bundle is globally parallelizable and $\tilde{A}$ is a scalar transformation.

Next example is a full minimal immersion of an $m$-dimensional sphere of curvature $m / 2(m+1)$ into a unit sphere of dimension $\{m+m(m+1) / 2-1\}$. Such an immersion is rigid, isotropic, and has a parallel second fundamental tensor ([6], [15]). We denote by $V^{m}$ the sphere $S^{m}(\sqrt{2(m+1) / m})$ together with this immersion and it is called the $m$-dimensional Veronese manifold. The mapping is explicitly constructed in terms of an orthonormal basis of harmonic polynomials of degree 2 and of $(m+1)$-variables. Since $S=m(m-1)(m+2) / 2(m+1)$ and $m S-K_{N}-L_{N}=0, \quad L_{N}=(1 / m) K_{N}=m^{2}(m-1)(m+2) / 2(m+1)^{2}$. The volume of $V^{m}$ is $(2(m+1) / m)^{m / 2} \omega_{m}$ where $\omega_{m}$ is the volume of the $m$-dimensional unit sphere. $V^{2}$ is the so-called Veronese surface and its explicit mapping is given by

$$
\begin{aligned}
& S^{2}(\sqrt{3}) \ni(x, y, z) \mapsto\left(u^{1}, \cdots, u^{5}\right) \in S^{4}, \\
& u^{1}=\frac{1}{\sqrt{3}} y z, \quad u^{2}=\frac{1}{\sqrt{3}} z x, \quad u^{3}=\frac{1}{\sqrt{3}} x y, \quad u^{4}=\frac{1}{2 \sqrt{3}}\left(x^{2}-y^{2}\right), \\
& u^{5}=\frac{1}{6}\left(x^{2}+y^{2}-2 z^{2}\right) .
\end{aligned}
$$

More generally, for each positive integer $k$, by an orthonormal basis of harmonic polynomials of degree $k$ and of three variables, we obtain a full minimal immer- 
sion of $S^{2}(\sqrt{k(k+1) / 2})$ into $S^{2 k}$. And such an immersion is rigid and is called the generalized Veronese surface of index $(k-1)([3])$. We denote by $V_{k-1}^{2}$ the surface $S^{2}(\sqrt{k(k+1) / 2})$ together with this immersion.

Now we state some lemmas which are needed in later sections. The following well known equality ([5]) is usefull.

$$
\frac{1}{2} \nabla^{2} S=m c S-K_{N}-L_{N}+T \text {. }
$$

Thus, $\int_{M}\left(m c S-K_{N}-L_{N}+T\right)=0$, from which we have

LEMMA 1.1. $\int_{M}\left(K_{N}+L_{N}\right) \geqq \int_{M} m c S$, where the equality holds if and only if the second fundamental tensor is parallel.

From this Lemma we know that in a space of constant negative curvature there exists no compact minimal submanifold with parallel second fundamental tensor except a totally geodesic one.

LEMMA 1.2. $\int_{M} K_{N} \leqq m \int_{M} L_{N}$, where the equality holds if and only if the ımmersion is isotropic and $M$ has a constant curvature ( $m \geqq 3)$.

$$
m c \int_{M} S \leqq(m+1) \int_{M} L_{N},
$$

where the equality holds if and only if the second fundamental tensor is parallel, the immersion is isotropic and $M$ has a constant curvature $(m \geqq 3)$.

proof. The former inequality is given in [13]. The latter can be obtained by this inequality and Lemma 1.1.

Q.E.D.

Now we make Kaehlerian preliminaries. Let $N=N(c)$ denote a complex $n$-dimensional Kaehler manifold of constant holomorphic curvature $c$. All complex submanifolds of $N$, which we consider, are assumed to be compact, connected, of class $C^{\infty}$, and to have no boundary. They are Kaehler manifolds by the induced metric and are minimal in $N$. Let $M$ be such a complex $m$-dimensional Kaehler submanifold of $N$. Let $J$ be the almost complex structure of $N$. The almost complex structure of $M$ is also denoted by $J$. Let $B$ be the Bochner curvature tensor of $M$, which is given by

$$
\begin{gathered}
B_{\imath j k l}=R_{\imath j k l}-\frac{1}{2 m+4}\left\{\rho_{j k} g_{\imath l}-\rho_{j l} g_{\imath k}+g_{j k} \rho_{\imath l}-g_{j l} \rho_{\imath k}+\sum_{r=1}^{2 m} \rho_{\jmath r} J_{k}^{r} J_{i l}-\rho_{\jmath r} J_{l} J_{\imath k}\right. \\
\left.\left.\quad+J_{j k} \rho_{\imath r} J_{l}^{r}-J_{j l} \rho_{\imath r} J_{k}^{r}-2 \rho_{k r} J_{l}^{r} J_{\imath \jmath}-2 \rho_{\imath r} J_{\jmath}^{r} J_{k l}\right)\right\} \\
+\frac{1}{4(m+1)(m+2)}\left(g_{j k} g_{i l}-g_{j l} g_{\imath k}+J_{\imath k} J_{\imath l}-J_{\jmath l} J_{\imath k}-2 J_{k l} J_{\imath \jmath}\right) \tau .
\end{gathered}
$$


Then $|B|^{2}$ is given by

$$
|B|^{2}=|R|^{2}-\frac{8}{m+2}|\rho|^{2}+\frac{2}{(m+1)(m+2)} \tau^{2} .
$$

The Kaehler manifold $M$ has a constant holomorphic curvature if and only if $|B|=0$ and $|G|=0$ hold $(m \geqq 2)$.

In the Kaehlerian case, $\widetilde{R}=-(m c / 2) I$ holds, thus the Jacobi differential operator becomes

$$
J=-\nabla^{2}-\frac{m c}{2} I-\tilde{A} .
$$

And the fundamental equality is $([21])$

$$
\frac{1}{2} \nabla^{2} S=T-\tilde{K}_{N}-L_{N}+\frac{m+2}{2} c S,
$$

where $\tilde{K}_{N}$ is defined by $\tilde{K}_{N}=\sum_{\imath, j, \lambda, \mu}\left(\sum_{k}\left(A_{j k}^{\lambda} A_{i k}^{\mu}-A_{\imath k}^{\lambda} A_{j k}^{\mu}\right)\right)^{2}$, and $A_{\imath \jmath}^{\lambda}$ 's are components of $A$. In the real case $K_{N}=\widetilde{K}_{N}$ holds, but in the Kaehlerian case $K_{N}=$ $m(n-m) c^{2}+2 c S+\widetilde{K}_{N}$, and $\widetilde{K}_{N}$ is commonly called the normal scalar curvature of the Kaehler submanifold.

LEMMA 1.3.

$$
\begin{aligned}
& \tilde{K}_{N}=(m+1)^{2} m c^{2}-2(m+1) c \tau+2|\rho|^{2}, \\
& L_{N}=m(m+1) c^{2}-2 c \tau+\frac{1}{2}|R|^{2} .
\end{aligned}
$$

Proof. Let $\left\{e_{1}, \cdots, e_{m}, e_{1 *}=J e_{1}, \cdots, e_{m *}=J e_{m}, e_{m+1}, \cdots, e_{n}, e_{(m+1) *}=J e_{m+1}, \cdots\right.$, $\left.e_{n *}=J e_{n}\right\}$ be an orthonormal frame of $N$ at a point such that $e_{a}$ and $e_{a^{*}}$ $(1 \leqq a \leqq m)$ are tangent to $M$. Let the ranges of indices $\imath, \jmath, k, l, ; \alpha, \beta$ and $\lambda, \mu$ be respectively such that $i, \jmath, k, l=1, \cdots, m, 1^{*}, \cdots, m^{*} ; \alpha, \beta=m+1, \cdots, n$ and $\lambda, \mu=m+1, \cdots, n,(m+1)^{*}, \cdots, n^{*}$. We write $A^{e_{\lambda}}$ simply by $A^{\lambda}$ and the components of $A^{\lambda}$ with respect to the above frame by $A_{i j}^{\lambda}$. First we note that $\sum A^{\lambda} A^{\mu} A^{\lambda} A^{\prime \prime}=0$, which follows from the observation that $\sum A^{\lambda} A^{\mu} A^{\lambda} A^{\prime \prime}=$ $\sum\left(A^{\alpha} A^{\beta} A^{\alpha} A^{\beta}+A^{\alpha} A^{\beta *} A^{\alpha} A^{\beta *}+A^{\alpha *} A^{\beta} A^{\alpha *} A^{\beta}+A^{\alpha *} A^{\beta *} A^{\alpha *} A^{\beta *}\right)$ and $J A^{\alpha}=-A^{\alpha} J, A^{\alpha *}$ $=J A^{\alpha}$. Thus

$$
\operatorname{trace}\left(\sum A^{\lambda} A^{\prime \prime} A^{\lambda} A^{\prime \prime}\right)=\sum A_{i j}^{\lambda} A_{k l}^{\lambda} A_{i l}^{\mu} A_{j k}^{\mu}=0 .
$$

Let $Q$ be the symmetric endomorphism of $T M$ corresponding to $\rho$ defined by $g(Q x, y)=\rho(x, y)$. Then $([21])$

$$
g(Q x, y)=\frac{m+1}{2} \operatorname{cg}(x, y)-2 \Sigma g\left(A^{\alpha} x, A^{\alpha} y\right) .
$$

Thus $Q=\frac{m+1}{2} c I-2 \Sigma\left(A^{\alpha}\right)^{2}$,

$$
4\left(\Sigma\left(A^{\alpha}\right)^{2}\right)^{2}=\frac{(m+1)^{2}}{4} c^{2} I-(m+1) c Q+Q^{2} .
$$


Taking the trace, we have

$$
4 \operatorname{tr}\left(\Sigma\left(A^{\alpha}\right)^{2}\right)^{2}=\frac{m(m+1)^{2}}{2}-c^{2}-(m+1) c \tau+|\rho|^{2} .
$$

On the other hand,

$$
\widetilde{K}_{N}=-\operatorname{tr} \sum\left(A^{\lambda} A^{\prime \prime}-A^{\prime \prime} A^{\lambda}\right)^{2}=8 \operatorname{tr}\left(\sum\left(A^{\alpha}\right)^{2}\right)^{2},
$$

Thus $\tilde{K}_{N}=m(m+1)^{2} c^{2}-2(m+1) c \tau+\left.\underset{\sim}{\mid} \rho\right|_{\sim} ^{2}$.

From the definition, $L_{N}=\operatorname{tr}(\tilde{A} \cdot \tilde{A})=\sum \tilde{A}_{\lambda \mu l} \tilde{A}_{\lambda \mu}=\sum A_{\imath \jmath}^{\lambda} A_{i j}^{\mu} A_{k l}^{\lambda} A_{k l}^{\mu}$. Let $\bar{R}$ be the curvature tensor of $N$. Then from the Gauss equation $R_{\imath j k l}=\bar{R}_{\imath j k l}-$ $\sum A_{j k}^{\lambda} A_{i l}^{\lambda}+\sum A_{i k}^{\lambda} A_{j l}^{\lambda}$, we obtain

$$
\sum R_{\imath j k l} R_{\imath j k l}=\sum \bar{R}_{\imath j k l} \bar{R}_{\imath \jmath k l}+\sum 4 A_{i k}^{\lambda} A_{j l}^{\lambda} \bar{R}_{\imath \jmath k l}+2 L_{N} .
$$

Since $\bar{R}_{\imath \jmath k l}=c / 4\left(-\delta_{\jmath k} \delta_{\imath l}+\delta_{j l} \delta_{\imath k}-J_{k}^{j} J_{l}^{i}+J_{k}^{i} J_{l}^{j}+2 J_{j}^{\imath} J_{l}^{k}\right), \sum A_{i k}^{\lambda} A_{\jmath l}^{\lambda} \delta_{j k} \delta_{\imath l}=S, \sum A_{i k}^{\lambda} A_{j l}^{\lambda} \delta_{\jmath l}$ $\delta_{i k}=0, \quad \sum A_{i k}^{\lambda} A_{j l}^{\lambda} J_{k}^{j} J_{l}^{i}=S, \quad \sum A_{i k}^{\lambda} A_{i l}^{\lambda} J_{k}^{i} J_{l}^{j}=0$, and $\sum A_{i k}^{\lambda} A_{j l}^{\lambda} J_{j}^{i} J_{l}^{k}=-S$, we obtain $\sum A_{\imath k}^{\lambda} A_{j l}^{\lambda} \bar{R}_{\imath j k l}=-c S$. On the other hand $\sum \bar{R}_{\imath j k l} \bar{R}_{\imath j k l}$ is equal to the value of the square norm of the curvature tensor of an $m$-dimensional complex space form with holomorphic curvature $c$. Then it is equal to $2 m(m+1) c^{2}$. Thus

$$
\begin{aligned}
& |R|^{2}=2 m(m+1) c^{2}-4 c S+2 L_{N}, \\
& L_{N}=m(m+1) c^{2}-2 c \tau+\frac{1}{2}|R|^{2} .
\end{aligned}
$$

Next we exhibit some of concrete Kaehler submanifolds in a complex projective space and state their characteristic properties.

Let $C P^{n}(c)$ denote the $n$-dimensional complex projective space of constant holomorphic sectional curvature $c(>0) . C P^{n}(1)$ is simply written as $C P^{n}$. Totally geodesic submanifolds of $C P^{n}$ are again complex projective spaces with the Fubini Study metric of holomorphic curvature 1.

Let $Q^{m}$ be the so-called $m$-dimensional complex quadratic. It is known that a compact hypersurface with constant scalar curvature immersed in $C P^{m+1}$ is $C P^{m}$ or $Q^{m}$ ([18]).

Next example is a Kaehler imbedding of $C P^{m}(1 / 2)$ into $C P^{m+m(m+1) / 2}$, which is a complex analogue of the Veronese manifold and has the same properties with it. Namely its second fundamental tensor is parallel and the imbedding is full and rigid ([21], Kaehler submanifolds of constant holomorphic curvature are necessarily isotropic). This Kaehler submanifold is called the m-dimensional complex Veronese manifold and we denote it by $C V^{m}$. The explicit construction of the imbedding is analogous to that of the real case ([23]).

$C V^{1}$ is identical with $Q^{1}$. When the dimension of the ambient space becomes higher, we have another example of rigid complex curve. That is, using homogeneous monomials of degree $n$ in homogeneous coordinates $\left(z_{0}, z_{1}, \cdots, z_{n}\right)$, we obtain an imbedding of $C P^{1}(1 / n)$ into $C P^{n}$;

$$
\left(z_{0}, z_{1}\right) \mapsto\left(z_{0}^{n} \sqrt{n} z_{0}^{n-1} z_{1}, \cdots, \sqrt{\frac{n !}{d !(n-d) !}} z_{0}^{n-d} z_{1}^{d}, \cdots, z_{1}^{n}\right) .
$$


Moreover, compact full complex curves of constant curvature in $C P^{n}$ is essentially unique and identical with the above one ([21]). We denote by $C V_{n-1}^{1}$ this complex curve together with this imbedding.

$\S 2$. Estimates of the first eigenvalue by means of geometrıc quantities

In this section we present some propositions concerning the estimates of the first eigenvalue of the Jacobi differential operator $J$ by means of geometric quantities.

For the first eigenvalue $\lambda_{1}$ of $J$, the minimum principle still holds. Namely, for a nonzero $C^{\infty}$ normal vector field $V$ on $M$,

$$
\lambda_{1} \leqq \begin{aligned}
& \int_{M}\langle J V, V\rangle \\
& \int_{M}|V|^{2}
\end{aligned}
$$

holds, and the equality is attained if and only if $V$ is a first eigenvector field. In other words, the first eigenvalue is the minimum value of the second variations of $M$ with respect to the normal variation vector fields of total norm 1 . In case $N$ is a space of constant curvature $c$, the inequality becomes

$$
\lambda_{1} \leqq-m c+\frac{\int_{M}\left(|\nabla V|^{2}-\left|A^{V}\right|^{2}\right)}{\int_{M}|V|^{2}} .
$$

First we treat such a case that $M$ is a hypersurface and there exists a global unit normal vector field $Y$ on $M$. Since such a $Y$ is parallel in the normal bundle, every normal vector field $V$ on $M$ has the form $V=f Y$, that is, there is a unique function $f$ on $M$ corresponding to $V$. Thus the minimum principle (2.2) becomes

$$
\lambda_{1} \leqq-m c+\frac{\int_{M}\left(|\nabla f|^{2}-S f^{2}\right)}{\int_{M} f^{2}}, \quad f \neq 0 \in C^{\infty}(M) .
$$

where the equality is attained if and only if $\int Y$ is a first eigenvector field of $J$. In general a normal vector field $V=\int Y$ is a $\lambda$-eigenvector field of $J$ i. e. $J V=\lambda V$, if and only if

$$
\nabla^{2} f=-(S+m c+\lambda) f \quad \text { on } M \text {. }
$$

Proposition 2.1. S is constant if and only if a first eigenvector jield is parallel $(n-m=1)$.

Proof. If a first eigenvector field $V=f Y$ is parallel, then $f$ is a nonzero constant and $0=\nabla^{2} f=-\left(S+m c+\lambda_{1}\right) f$, thus $S+m c+\lambda_{1}=0$ on $M$. Then $S$ is con- 
stant. The converse is also obvious.

Q.E. D.

Proposition 2.2. For a minımal hypersurface $M$ of $N$

$$
\lambda_{1} \leqq-m c-\frac{\int_{M} S}{\operatorname{vol}(M)}
$$

holds, and the equality is attained if and only if $S$ is constant. And $\lambda_{1}=-m c$ holds if and only if $M$ is totally geodesic.

Proof. Let $f$ be a nonzero constant in (2.3), then we obtain the inequality. Conversely if $\lambda_{1}=-m c-S(M) / \operatorname{vol}(M)$ holds, then any nonzero parallel normal vector field is a first eigenvector field, thus from Proposition 2.1, $S$ is constant. The last statement of the Proposition is obvious.

Q.E.D.

Proposition 2.3. For a non-totally geodesic mmmal hypersurface $M$

$$
\lambda_{1} \leqq-2 m c-\frac{\int_{M}\left(2 S T-|\nabla S|^{2}\right)}{2 \int_{M} S^{2}}
$$

holds.

Proof. From the assumption, $S \neq 0$. Putting $f=S$ in (2.3), and using the equality $\nabla^{2} S=2 m c S-2 S^{2}+2 T$, we obtain the desired inequality Q.E.D.

The following result is a restatement of Lemma 6.1 .7 given in [24].

Proposition 2.4. For a non-totally geodesic mmmal hypersurface $M, \lambda_{1} \leqq$ $-2 m$ holds ( $2 n$ case $N=S^{n}$ ).

In the case of an arbitrary codimension, we have the following results.

Proposition 2.5. $-m c-\max S \leqq \lambda_{1}$. The equality is attained if and only if the first eigenvector fields are parallel, and $M$ is totally geodesic or m-index of $M$ is everywhere equal to 1.

Proof. Let $V$ be a $\lambda_{1}$-eigenvector field of $J$. Then

$$
0 \leqq|\nabla V|^{2}(M)=\left(\lambda_{1}+m c\right)|V|^{2}(M)+\langle\tilde{A} V, V\rangle(M) \text {. }
$$

Thus $-\lambda_{1}-m c \leqq \frac{\langle\tilde{A} V, V\rangle(M)}{|V|^{2}(M)} \leqq \frac{S|V|^{2}(M)}{|V|^{2}(M)} \leqq \max _{x \in M} S(x)$. If the equality holds in these inequalities, then $V$ is parallel, so that $S$ is constant, $\tilde{A} V=S V$ holds, and $\operatorname{rank} \tilde{A}=0$ or $1 . \operatorname{rank} \tilde{A}=1$ on $M$ is equivalent to that its $m$-index is equal to 1 . Thus these conditions are summerized as in the statement of the Proposition.

Q.E.D. 
COROLlary 2.6. Let $M^{m}$ be a minımal submanifold of $S^{n}$. If $S \leqq m$ and $\lambda_{1} \leqq-2 m$ hold, then $M$ is an m-dimensional Clifford hypersurface in an $(m+1)$ dimensional totally geodesic submanifold $S^{m+1}$ of $S^{n}$.

Proof. From the assumption, $\max S=m$ and $-m-\max S=\lambda_{1}$. Thus $m$-index of $M$ is everywhere equal to 1 and then by a theorem of Otsuki ([22]) there exists an $(m+1)$-dimensional totally geodesic submanifold $S^{m+1}$ of $S^{n}$ in such a way that $M$ is contained and minimal in the $S^{m+1}$. Thus from [5] the statement follows.

Q.E. D.

Here we introduce two continuous functions $P_{0}, P_{1}$ on $M$. Let $x$ be an arbitrary point of $M$. Then $P_{0}(x)$ (resp. $P_{1}(x)$ ) is defined by the minimum (resp. maximum) value of sum of square principal curvature with respect to all normal directions at $x$, or equivalently $P_{0}(x)$ (resp. $P_{1}(x)$ ) is defined by the minimum (resp. maximum) eigenvalue of the symmetric endomorphism $\tilde{A}(x)$ of the normal space $T M_{. r}^{\perp}$. They satisfy the inequality; $P_{0} \leqq S / n-m \leqq P_{1}$, and in either side the equality is attained if and only if $\tilde{A}$ is a global scalar transformation (for example in case $M$ is of codimension 1 ).

Let $\left\{(0 \leqq) \lambda_{1}^{0} \leqq \lambda_{2}^{0} \leqq \cdots\right\}$ denote the complete set of eigenvalues of $-\nabla^{2}$ acting on normal vector fields on $M ;-\nabla^{2} V=\lambda V$. Note that $\lambda_{1}^{0}=0$ holds if and only if there exists a nonzero parallel normal vector field. Note also that in case $M$ is of codimension 1 , and $M$ and $N$ are both orientable, $\left\{\lambda_{1}^{0}, \lambda_{2}^{0}, \lambda_{3}^{0}, \cdots\right\}$ is an intrinsic invariant of $M$ while $\operatorname{Spec}(M, N)$ is in general an extrinsic invariant. By making use of the Courant's mini-max principle, we obtain a comparison relation between $\lambda_{k}$ 's and $\lambda_{k}^{\prime}$ 's.

Proposition 2.7. For each $k \geqq 1$

$$
-m c-\max P_{1}+\lambda_{k}^{0} \leqq \lambda_{k} \leqq-m c-\min P_{0}+\lambda_{k}^{0} .
$$

If $\tilde{A}$ is a scalar transformation,

$$
-m c-\max \frac{S}{n-m}+\lambda_{k}^{0} \leqq \lambda_{k} \leqq-m c-\min \frac{S}{n-m}+\lambda_{k}^{0}
$$

holds. In partıcular if $\tilde{A}$ is a scalar transformation and $S$ is constant,

$$
\lambda_{k}=\lambda_{k}^{n}-m c-\frac{S}{n-m} .
$$

Proof. Take $(k-1)$-arbitrary $C^{\infty}$ normal vector fields $V_{1}, \cdots, V_{k-1}$ and set

$$
\begin{array}{r}
M n\left(V_{1}, \cdots, V_{k-1}\right):=\inf \left\{\langle\langle J W, W\rangle\rangle \mid W ; C^{\infty} \text { normal field, }\|W\|=1,\right. \\
\left.\left\langle\left\langle W, V_{\imath}\right\rangle\right\rangle=0(1 \leqq \imath \leqq k-1)\right\}, \\
M n^{0}\left(V_{1}, \cdots, V_{k-1}\right):=\inf \left\{\left\langle\left\langle-\nabla^{2} W, W\right\rangle\right\rangle \mid W ; C^{\infty} \text { normal field, }\|W\|=1,\right. \\
\left.\left\langle\left\langle W, V_{\imath}\right\rangle\right\rangle=0(1 \leqq \imath \leqq k-1)\right\},
\end{array}
$$


where $\langle\langle\rangle$,$\rangle and \|\|$ denote the global inner product and norm. Then

$$
\begin{aligned}
& \lambda_{k}=\operatorname{Max}\left\{\operatorname{Mn}\left(V_{1}, \cdots, V_{k-1}\right) \mid V_{1}, \cdots, V_{k-1} ; C^{\infty} \text { normal fields }\right\}, \\
& \lambda_{k}^{0}=\operatorname{Max}\left\{\operatorname{Mn}^{0}\left(V_{1}, \cdots, V_{k-1}\right) \mid V_{1}, \cdots, V_{k-1} ; C^{\infty} \text { normal fields }\right\} .
\end{aligned}
$$

Since $\langle\langle J W, W\rangle\rangle=\left\langle\left\langle-\nabla^{2} W, W\right\rangle\right\rangle-m c-\langle\langle\tilde{A} W, W\rangle\rangle$ for a $W$ satisfying the above condition,

$$
-m c-\max P_{1}+\left\langle\left\langle-\nabla^{2} W, W\right\rangle\right\rangle \leqq\langle\langle J W, W\rangle\rangle \leqq-m c-\min P_{0}+\left\langle\left\langle-\nabla^{2} W, W\right\rangle,\right.
$$

holds. Thus

$$
\begin{aligned}
& -m c-\max P_{1}+M n^{0}\left(V_{1}, \cdots, V_{k-1}\right) \leqq M n\left(V_{1}, \cdots, V_{k-1}\right) \leqq-m c \\
& -\min P_{0}+M n^{0}\left(V_{1}, \cdots, V_{k-1}\right),
\end{aligned}
$$

and $-m c-\max P_{1}+\lambda_{k}^{0} \leqq \lambda_{k} \leqq-m c-\min P_{0}+\lambda_{k}^{0}$.

Q.E. D.

PROPOSITION 2.8.

(1). If $\lambda_{1}<-m c-\min P_{1}$ or $-m c-\max P_{0}<\lambda_{1}$, then no first eigenvector fields are parallel.

(2). Let $N$ be $S^{n}$. If $\tilde{A}$ is a scalar transformation, $S$ constant, and $-m-m / 2(n-m)-1+\lambda_{1}^{0} \leqq \lambda_{1}$ holds, then $M$ is a Veronese surface in $S^{1}$, or an $m$-dimensional Ciifford hypersurface in $S^{m+1}$, or a totally geodesic $S^{m}$ in $S^{n}$.

Proof. (1). Suppose that there exists a parallel first eigenvector field $V$. Then $\tilde{A} V=-\left(\lambda_{1}+m c\right) V$, thus $P_{0}\langle V, V\rangle \leqq-\left(\lambda_{1}+m c\right)\langle V, V\rangle \leqq P_{1}\langle V, V\rangle$. Since $V$ nowhere vanishes, $\max P_{0} \leqq-\lambda_{1}-m c \leqq \min P_{1}$, i. e.

$$
-m c-\min P_{1} \leqq \lambda_{1} \leqq-m c-\max P_{0} \text {. }
$$

(2). From Proposition 2.7, $S \leqq m /(2-1 / n-m)$. Thus from [5] the conclusion follows.

Q.E. D.

In general the number of negative eigenvalues in $\operatorname{Spec}(M, N)$ is the so-called index of the minimal submanifold $M$, and the multiplicity of the zero eigenvalue is the so-called nullity. And 0-eigenvector fields are so-called Jacobi fields on $M$. This is the reason why we call the operator $J$ the Jacobi differential operator. Any totally geodesic submanifold of $S^{n}$ is characterized by its index or nullity. The following two theorems are due to J. Simons ([24]).

THEOREM 2.9 Every $\operatorname{Spec}\left(M^{m}, S^{n}\right)$ contams $-m$ at least $(n-m)$-tmmes and just $(n-m)$-trmes when and only when $M$ is totally geodesic.

TheOREM 2.10 Every $\operatorname{Spec}\left(M^{m}, S^{n}\right)$ contams 0 at least $(m+1)(n-m)$-trmes and Just $(m+1)(n-m)$-trmes when and only when $M$ is totally geodesic.

In the Kaehlerian case we have following theorem due to Y. Kimura ([17]).

THeorem 2.11. For a compact Kaehler submanfold $M^{m}$ of $C P^{n}, \lambda_{1}=0$ holds. 
And its multiplicity $\geqq 2(m+1)(n-m)$, where the equality holds when and only when $M$ is totally geodesic.

\section{$\S 3$. Review of a Gilkey's paper.}

In this section we review the Gilkey's paper [10]. Let $M$ be a compact connected Riemannian manifold of dimension $m, g$ its Riemannian metric, $v_{g}$ its canonical measure. Let $V$ be a smooth vector bundle over $M$ and $D ; C^{\infty}(V) \rightarrow$ $C^{\infty}(V)$ a second order differential operator with leading symbol given by the metric tensor. Locally $D$ can be expressed as

$$
D=-\left(\sum g^{i \jmath} \frac{\partial^{2}}{\partial x_{i} \partial x_{\jmath}}+\sum M_{k} \frac{\partial}{\partial x_{k}}+N\right),
$$

where $\left(x_{\imath}\right)_{(1 \leqq \imath \leqq m}$ is a local coordinate system and $M_{k}$ and $N$ are square matrices which depend on the choice of frame and local coordinates.

Let $V_{X}$ denote the fibre of $V$ at $x$. For $t>0, \exp (-t D)$ is a well defined infinitely smoothing operator which is of trace class in $L^{2}(V)$. Let $K(t, x, y, D)$ be the kernel of $\exp (-t D)$, which is a homomorphism from $V_{y}$ to $V_{x}$. Then

$$
\exp (-t D) u(x)=\int_{M} K(t, x, y, D) u(y) v_{g}(y)
$$

It is well-known that as $t \downarrow 0$, Trace $K(t, x, x, D)$ has a uniform asymptotic expansion of the form

$$
\operatorname{Tr} K(t, x, x, D) \sim \sum_{k=0}^{\infty} B(x, D) t^{k-m / 2} .
$$

The coefficients $B_{k}(x, D)$ are smooth functions of $x$ which can be computed functorially in terms of the derivatives of the total symbol of the differential operator $D . B_{k}(x, D)$ is a local invariant of $D$ and $B_{2 k+1}(x, D)=0$. Set $B_{k}(D)$ $=\int_{M} B_{k}(x, D) V_{g}(X)$. Then

$$
\int_{M} \operatorname{Tr} K(t, x, x, D) \underset{t \downarrow 0}{\sim} \sum_{k=0}^{\infty} B_{k}(D) t^{k-m / 2}
$$

If $V$ has a smooth inner product $\langle$,$\rangle on each fibre and if D$ is self-adjoint with respect to the fibre metric, let $\left\{\lambda_{\nu}, \theta_{\nu}\right\}_{\nu=1}^{\infty}$ be a complete spectral decomposition of $D$ into an orthonormal basis of eigensections $\theta_{\nu}$ and corresponding eigenvalues $\lambda_{\nu}$. For such a $D$, we can express

$$
\begin{aligned}
& \operatorname{Tr} K(t, x, x, D)=\sum_{\nu=1}^{\infty} \exp \left(-t \lambda_{\nu}\right)\left\langle\theta_{\nu}, \theta_{\nu}\right\rangle(x), \\
& \int_{M} \operatorname{Tr} K(t, x, x, D)=\sum_{\nu=1}^{\infty} \exp \left(-t \lambda_{\nu}\right) .
\end{aligned}
$$

Thus the integrated invariants $B_{k}(D)$ depend only on the asymptotic behavior of the partition function $\Sigma \exp \left(-t \lambda_{\nu}\right)$ and therefore spectral invariants.

Let $\nabla$ be the Levi-Civita connection on $T M$ and also let $\nabla$ be any connection 
on the vector bundle $V$. Let $W$ be the curvature tensor of the connection $\nabla$ on $V$. Let $\nabla^{2}$ be the Laplace operator on $V$ defined by $\nabla$ and $g$.

THEOREM 3.1 ([9]) Given a second order differential operator $D ; C^{\infty}(V) \rightarrow$ $C^{\infty}(V)$ with leading symbol given by the metruc tensor, there is a unique connection $\nabla$ on $V$ such that $E:=-\nabla^{2}-D$ is a 0 th order operator, i.e. an endomorphism of $V$.

Then for a $D$ we consider only such a connection $\nabla$ on $V$. Let $r$ be the fibre dimension of $V$ and set $A_{k}(D)=(4 \pi)^{m / 2} B_{2 k}(D)$. (We note here that the signs of $R, \rho$ and $\tau$ used in [10] are different from ours). Then

$$
\Sigma \exp \left(-t \lambda_{\nu}\right) \underset{t \downarrow 0}{\sim}(4 \pi t)^{-m / 2} \cdot\left(A_{0}(D)+A_{1}(D) t+A_{2}(D) t^{2}+\cdots\right) .
$$

THEOREM $3.2([9],[10])$

(1). $. A_{0}(D)=r \cdot \operatorname{vol}(M)$,

(2). $\quad A_{1}(D)=\frac{\gamma}{6} \int_{M} \tau+\int_{M} \operatorname{Tr}(E)$

(3). $A_{2}(D)=\frac{\gamma}{360} \int_{M}\left(5 \tau^{2}-2|\rho|^{2}+2|R|^{2}\right)+\frac{1}{360} \int_{M}\left\{-30|W|^{2}+\operatorname{Tr}\left(60 \tau E+180 E^{2}\right)\right\}$.

\section{$\S 4$. Asymptotic expansion of the partition function.}

In this section we apply the results stated in $\S 3$ to our case and clarify the geometric meaning of the first three terms of the asymptotic expansion for $t \downarrow 0$ of the partition function $\sum_{\imath=1}^{\infty} e^{-\lambda_{i} t}$, and then obtain Riemannian and Kaehlerian spectral invariants. The vector bundle $V$ considered in $\S 3$ is in our case replaced by the normal bundle $T M^{\perp}$ of the minimal submanifold $M$, which has the canonical inner product. Thus the fibre dimension of $V$ is just the codimension of $M$. The differential operator $J$ consists of the Laplace operator $\nabla^{2}$ of the normal bundle and its symmetric endomorphism $(-\widetilde{R}+\tilde{A})$. Thus to the endomorphism $E$ considered in $\S 3$ corresponds the endomorphism $(-\widetilde{R}+\tilde{A})$ and the required unique connection on $T M^{\perp}$ is just the normal connection induced from the immersion. Then from the Gauss equation,

$$
\begin{aligned}
& \tau=m(m-1) c-S, \quad \operatorname{Tr}(E)=S+m(n-m) c, \\
& \operatorname{Tr}\left(E^{2}\right)=L_{N}+2 m c S+m^{2}(n-m) c^{2} .
\end{aligned}
$$

Let $\operatorname{Spec}(M, N)$ be $\left\{\lambda_{1}, \lambda_{2}, \cdots\right\}$. Let us express the asymptotic expansion of $\sum e^{-\lambda_{i} t}$ for $t \downarrow 0$ in the way;

$$
\sum e^{-\lambda_{i} t} \sim(4 \pi t)^{-m / 2} \cdot\left(a_{0}+a_{1} t+a_{2} t^{2}+\cdots\right) .
$$

Then each $a$, is an integration over $M$ of a local invariant determined by the total symbol of $J$ which is expressed by the second fundamental tensor $A$ and 
the metric tensor $g$. And derivatives of $g$ are expressed via E. Cartan's theorem by covariant derivatives of the curvature tensor $R$, and $R$ itself is expressed through the Gauss equation in terms of $A$. Consequently each $a_{\jmath}$ is an integration over $M$ of a local invariant determined by the second fundamental tensor and its higher order covariant derivatives.

From Theorem 3.2, we have

THEOREM 4.1. Let $M^{m}$ be a minimal submanifold of $N^{n}(c)$. Then

$$
\begin{aligned}
a_{0}= & (n-m) \cdot \operatorname{vol}(M), \\
a_{1}= & m(n-1) c \cdot \operatorname{vol}(M)+\frac{n-m-6}{6} \int_{M} \tau \\
= & \frac{m}{6}(m+5)(n-m) c \cdot \operatorname{vol}(M)-\frac{n-m-6}{6} \int_{M} S, \\
a_{2}= & \frac{n-m}{360} \int_{M}\left(5 \tau^{2}-2|\rho|^{2}+2|R|^{2}\right)+\frac{1}{12} \int_{M}\left(6 L_{N}-K_{N}\right) \\
& +\frac{1}{6} \int_{M}\left(m(n-7) c \cdot \tau-\tau^{2}\right)+C_{0}(m, n, c) \cdot \operatorname{vol}(M),
\end{aligned}
$$

where $C_{0}(m, n, c)$ is a number determmed by $m, n$ and $c$.

Corollary 4.2. Let $M$ be a minımal submanifold of a given space $N$ of constant curvature $c$. If the codimension of $M$ is not equal to 6 , then the spectrum $\operatorname{Spec}(M, N)$ determines the following quantities (spectral invariants);

(1). dimension of $M$, volume of $M, \int_{M} \tau, \int_{M} S, \int_{M}\left(K_{N}+L_{y}-T\right)$,

(2). $\quad \frac{n-m}{180} \int_{M}\left(|R|^{2}-|\rho|^{2}\right)+\frac{n-m-12}{72} \int_{M} \tau^{2}+\frac{1}{12} \int_{M}\left(6 L_{N}-K_{N}\right)$,

(3). $\quad \frac{n-m}{180} \int_{M}\left(|C|^{2}+\frac{6-m}{m-2}|G|^{2}\right)+C_{1}(m, n) \int_{M} \tau^{2}+\frac{1}{12} \int_{M}\left(6 L_{N}-K_{N}\right)$,

(4). $\frac{n-m}{180} \int_{M}\left(|C|^{2}+\frac{6-m}{m-2}|G|^{2}\right)+C_{1}(m, n) \int_{M} \tau^{2}+\frac{1}{12} \int_{M}\left(6 T_{N}-7 K_{N}\right)$,

where $C_{1}(m, n)=\frac{-5 m^{3}-53 m^{2}+54 m+\left(5 m^{2}-7 m+6\right) n}{360 m(m-1)}$.

We note that if $2 \leqq m$ and $n \geqq m+13$, then $C_{1}(m, n)>0$, and if $4 \leqq m$ and $n \leqq m+12$, then $C_{1}(m, n)<0$.

Proposition 4.3. For an oriented 4-dimensional minimal submanifold $M$, let $\chi(M)$ and $\operatorname{sign}(M)$ denote the Euler number and signature of $M$, then the following are its spectral invariants. 


$$
\begin{aligned}
\frac{2(n-4)}{5} \pi^{2} \cdot \operatorname{sign}(M) & +\frac{4(n-4)}{45} \pi^{2} \cdot \chi(M)-\left(C_{1}(4, n)+\frac{7(n-4)}{2160}\right) \int_{M} \tau^{2} \\
& -\frac{1}{12} \int_{M}\left(6 L_{N}-K_{N}\right), \\
\frac{2(n-4)}{5} \pi^{2} \cdot \operatorname{sign}(M) & +\frac{4(n-4)}{45} \pi^{2} \cdot \chi(M)-\left(C_{1}(4, n)+\frac{7(n-4)}{2160}\right) \int_{M} \tau^{2} \\
& -\frac{1}{12} \int_{M}\left(6 T-7 K_{N}\right) .
\end{aligned}
$$

Proof. From the generalized Gauss-Bonnet formula and the Hirzebruch signature theorem, $\chi(M)$ and $\operatorname{sign}(M)$ can be expressed in terms of $|C|,|G|$ and $\tau$ as $([7])$

$$
\chi(M)=\frac{1}{32 \pi^{2}}\left(|C|^{2}-2|G|^{2}+\frac{1}{6} \tau^{2}\right)(M), \quad \operatorname{sign}(M)=\frac{-1}{48 \pi^{2}}\left(|C|^{2}-\frac{1}{3} \tau^{2}\right)(M) .
$$

Thus

$$
\begin{aligned}
|C|^{2}(M) & =32 \pi^{2} \cdot \chi(M)+\left(2|G|^{2}-\frac{1}{6} \tau^{2}\right)(M) \\
& =\frac{1}{3} \tau^{2}(M)-48 \pi^{2} \cdot \operatorname{sign}(M) .
\end{aligned}
$$

Substituting these into (3) of Corollary 4.2, we obtain two spectral invariants;

$$
\begin{aligned}
& -\frac{4(n-4)}{15} \pi^{2} \cdot \operatorname{sign}(M)+\frac{n-4}{180} \int_{M}|G|^{2}+\left(C_{1}(4, n)+\frac{n-4}{540}\right) \int_{M} \tau^{2}+\frac{1}{12} \int_{M}\left(6 L_{Y}-K_{N}\right), \\
& \frac{8(n-4)}{45} \pi^{2} \cdot \chi(M)+\stackrel{n-4}{60} \int_{M}|G|^{2}+\left(C_{1}(4, n)-\begin{array}{l}
n-4 \\
1080
\end{array}\right) \int_{M} \tau^{2}+\frac{1}{12} \int_{M}\left(6 L_{N}-K_{N}\right)
\end{aligned}
$$

Eliminating $|G|$ from these, we obtain the desired first invariant. The second invariant can be obtained in the same way, using (4) of Corollary 4.2.

Q E.D.

Now let $N=N(c)$ be an $n$-dimensional Kaehler manifold of constant holomorphic sectional curvature $c$, and $M$ an $m$-dimensional Kaehler submanifold of $N$. Then from the Gauss equation

$$
\tau=m(m+1) c-S,
$$

and from $E=(m / 2) c I+\tilde{A}$ and Lemma 1.3

$$
\begin{aligned}
& \operatorname{tr}(E)=m(n-m) c+S=m(n+1) c-\tau, \\
& \operatorname{tr}\left(E^{2}\right)=\frac{(n-m) m^{2}}{2} c^{2}+m c S+L_{N}
\end{aligned}
$$




$$
=\frac{m}{2}\left(m^{2}+m n+4 m+2\right) C^{2}-(m+2) c \tau+\frac{1}{2}|R|^{2} .
$$

THEOREM 4.4. Let $M^{n}$ be a Kaehler submanifold of $N^{n}(c)$. Then

$$
\begin{aligned}
a_{0}= & 2(n-m) \cdot \operatorname{vol}(M), \\
a_{1}= & (n+1) m c \cdot \operatorname{vol}(M)+\frac{n-m-3}{3} \int_{M} \tau \\
= & \frac{(n-m)(m+4)}{3} m c \cdot \operatorname{vol}(M)-\frac{n-m-3}{3} \int_{M} S, \\
a_{2}= & \int_{M}\left\{\left(\frac{n-m}{90}+\frac{1}{4}\right)|R|^{2}-\left(\frac{n-m}{90}+\frac{1}{6}\right)|\rho|^{2}+\left(\frac{n-m}{36}-\frac{1}{6}\right) \tau^{2}\right\} \\
& +C_{2}(m, n, c) \int_{M} \tau+C_{3}(m, n, c) \cdot \operatorname{vol}(M),
\end{aligned}
$$

where $C_{2}(m, n, c)$ and $C_{3}(m, n, c)$ are numbers determmed by $m, n$ and $c$.

COROLlARY 4.5. Let $M$ be a Kaehler submannfold of a given complex space form $N$. If the complex codimension of $M$ is not equal to 3 , then the following are its spectral invariants.

(1). dimension of $M$, volume of $M, \int_{M} \tau, \int_{M} S$,

(2). $\int_{M}\left\{\left(\frac{n-m}{90}+\frac{1}{4}\right)|R|^{2}-\left(\frac{n-m}{90}+\frac{1}{6}\right)|\rho|^{2}+\left(\frac{n-m}{36}-\frac{1}{6}\right) \tau^{2}\right\}$,

(3). $\int_{M}\left\{\frac{2(n-m)+45}{180}|B|^{2}+\frac{(n-m)(6-m)-15 m+150}{90(m+2)}|G|^{2}+C_{4}(m, n) \tau^{2}\right\}$,

(4). $\int_{M}\left\{\frac{1}{2}|B|^{2}+\frac{2 m+8}{m+2}|G|^{2}+\frac{m+2}{m(m+1)} \tau^{2}-T\right\}$,

(5). $\left.\int_{M}\left\{\frac{n-m}{18}+\frac{7}{6}\right)|G|^{2}+\frac{(1-m)(n-m)+6 m+21}{36 m} \tau^{2}-\left(\frac{n-m}{45}+\frac{1}{2}\right) T\right\}$, where $C_{4}(m, n)=\frac{\left(5 m^{2}+4 m+3\right)(n-m)-30 m^{2}-45 m+75}{180 m(m+1)}$.

Proof. (1) and (2) are obviously spectral invariants. Substituting $|\rho|^{2}=$ $|G|^{2}+\frac{1}{2 m} \tau^{2}$ and $|R|^{2}=|B|^{2}+\frac{8}{m+2}|G|^{2}+\frac{2}{m(m+1)} \tau^{2}$ into (2), we obtain (3). Integrating both sides of the equality $\frac{1}{2} \nabla^{2} S=T-\widetilde{K}_{N}-L_{N}+\frac{m+2}{2} c S$, and using (1) and Lemma 1.3, we obtain (4). Eliminating $B$ from (3) and (4), we obtain (5).

Q.E. D. 
Assumption. In the following we consider only such minimal submanifolds that their (real) codimensions are not equal to 6 .

\section{$\S 5$. Geometry reflected by the spectrum.}

In this section, making use of the spectral invariants given in $\S 4$, we obtain some properties which are derived from or reflected under the isospectral condition. These properties consist mainly of three parts, i. e. constant curvature property, Einstein property and the miscellaneous one, together with isotropic or parallel second fundamental tensor property. Then using these isospectral properties we characterize some concrete minimal submanifolds of a sphere, particularly Veronese manifolds.

First we state those which are related with constant curvature property.

Proposition 5.1. For a minmal surface $M$, its Euler number $\chi(M)$ and the integral $\int_{M}\left\{(n+18) S^{2}-20 K_{N}\right\}$ are spectral mvariants. And the followng are spectral properties.

(1). The immersion is isotropic and $M$ has a constant Gaussian curvature K.

(2). $M$ has a positıve constant Gaussian curvature K.

Proof. For a surface $M,|R|^{2}=2|\rho|^{2}=\tau^{2}$ and $2 L_{N}=2 S^{2}-K_{N}$ hold. Thus from Corollary 4.2, $\left\{(n+18) S^{2}-20 K_{N}\right\}(M)$ is a spectral invariant. By the BaussBonnett formula $\chi(M)$ is also a spectral invariant. When $M$ is of dimension 2, $S^{2}(M) \geqq K_{N}(M)$ holds, and the equality is attained if and only if the immersion is isotropic. Now let $M$ and $M^{\prime}$ be minimal surfaces of $N$ with the same spectrum. $M$ is assumed to be isotropic and of constant Gaussian curvature $K$. Then

$$
\begin{aligned}
& \left((n+18) S^{2}-20 K_{N}\right)(M)=\left((n+18) S^{\prime 2}-20 K_{N}^{\prime}\right)\left(M^{\prime}\right), \\
& K_{N}(M) \geqq K_{N}^{\prime}\left(M^{\prime}\right), \quad S^{2}(M) \geqq S^{\prime 2}\left(M^{\prime}\right) .
\end{aligned}
$$

Since $S$ is constant and area $(M)=\operatorname{area}\left(M^{\prime}\right)$, by the Schwarz inequality

$$
\operatorname{area}(M) \cdot S^{2}(M)=(S(M))^{2}=\left(S^{\prime}\left(M^{\prime}\right)\right)^{2} \leqq \operatorname{area}(M) \cdot S^{\prime 2}\left(M^{\prime}\right) .
$$

Thus $S^{2}(M) \leqq S^{\prime 2}\left(M^{\prime}\right), S^{2}(M)=S^{\prime 2}\left(M^{\prime}\right)$, and $S=S^{\prime}=$ constant, $K_{N}(M)=K_{N}^{\prime}\left(M^{\prime}\right)$ hold. Then $S^{\prime 2}\left(M^{\prime}\right)=K_{N}^{\prime}\left(M^{\prime}\right)$ and (1) follows. (2) follows immediately from (1), because a minimal surface with positive Gaussian curvature is isotropic ([12]). Q. E.D.

COROLlary 5.2. If a full minmal submanifold $M$ of $S^{2 n}$ has the same spectrum with the generalized Veronese surface $V_{n-1}^{2}$, then $M$ is itself $V_{n-1}^{2}$.

Proof. From the assumption and Proposition 5.1, $M$ is a surface of constant Gaussian curvature $2 / n(n+1)$, and the area of which is equal to that of $S^{2}(\sqrt{n(n+1) / 2)}$. Thus if the immersion is full, it is the generalized Veronese surface $V_{n-1}^{2}$.

Q.E. D. 
COROLlary 5.3. The Clifford torus $M_{1,1}$ is characterzed by its spectrum.

Proof. For a minimal surface $M$ in $S^{3}, \tau^{2}(M)$ is a spectral invariant. Thus the Gaussian curvature being a constant $K$ is a spectral property. In particular, if $\operatorname{Spec}\left(M, S^{3}\right)=\operatorname{Spec}\left(M_{1,1}, S^{3}\right)$ holds, $M$ is also flat and $\operatorname{area}(M)=\operatorname{area}\left(M_{1,1}\right)$, thus $M=M_{1,1}([4])$.

Q.E.D.

Proposition 5.4. If $2 \leqq m \leqq 5$ and $n \geqq m+13$, then the followng is a spectral property, The immersion is isotropic, the second fundamental tensor parallel and the sectional curvature a constant $k$.

Proof. Suppose that $\operatorname{Spec}(M, N)=\operatorname{Spec}\left(M^{\prime}, N\right), \quad 2 \leqq m \leqq 5$, and $n \geqq m+13$. Assume that $M$ is isotropic and has a constant curvature $k$. Suppose that $\nabla A$ $=0$ holds. Then from Lemma 1.2 (in case $m \geqq 3$ )

$$
(m+1) L_{N}(M)=m c S(M)=m c S^{\prime}\left(M^{\prime}\right) \leqq(m+1) L_{N}^{\prime}\left(M^{\prime}\right),
$$

thus $L_{N}(M) \leqq L_{N}^{\prime}\left(M^{\prime}\right)$, and from Corollary 4.2

$$
\begin{aligned}
& C_{1}(m, n) \tau^{2}(M)+\frac{1}{12}(6-m) L_{N}(M) \\
& =\frac{n-m}{180}\left(\left|C^{\prime}\right|^{2}+\frac{6-m}{m-2}\left|G^{\prime}\right|^{2}\right)\left(M^{\prime}\right)+C_{1}(m, n) \tau^{\prime 2}\left(M^{\prime}\right)+\frac{1}{12}\left(6 L_{N}^{\prime}-K_{N}^{\prime}\right)\left(M^{\prime}\right) \\
& \geqq C_{1}(m, n) \tau^{\prime 2}\left(M^{\prime}\right)+\frac{1}{12}\left(6 L_{N}^{\prime}-K_{N}^{\prime}\right)\left(M^{\prime}\right) \\
& \geqq C_{1}(m, n) \tau^{\prime 2}\left(M^{\prime}\right)+\frac{1}{12}(6-m) L_{N}^{\prime}\left(M^{\prime}\right) \\
& \geqq C_{1}(m, n) \tau^{\prime 2}\left(M^{\prime}\right)+\frac{1}{12}(6-m) L_{N}(M) .
\end{aligned}
$$

From $C_{1}(m, n)>0, \quad \tau^{2}(M) \geqq \tau^{\prime 2}\left(M^{\prime}\right)$ follows. And $\tau^{2}(M)=\tau^{\prime 2}\left(M^{\prime}\right)$ and $\tau=\tau^{\prime}$ hold because $\tau$ is constant and $\tau(M)=\tau^{\prime}\left(M^{\prime}\right)$. Therefore in the above, all inequalities become equalities. Thus

$$
\left|C^{\prime}\right|=0, \quad\left|G^{\prime}\right|=0, \quad\left(m L_{N}^{\prime}-K_{N}^{\prime}\right)\left(M^{\prime}\right)=0, \quad m c S^{\prime}\left(M^{\prime}\right)=(m+1) L_{N}^{\prime}\left(M^{\prime}\right) .
$$

It follows that $M$ is isotropic, has a constant curvature $k$ and that $\nabla A^{\prime}=0$. In the case of $m=2$, by Proposition $5.1, K_{N}(M)=K_{N}^{\prime}\left(M^{\prime}\right)$ and $S^{2}(M)=S^{\prime 2}\left(M^{\prime}\right)$ hold, thus $L_{N}(M)=L_{N}^{\prime}\left(M^{\prime}\right)$, and from Lemma 1.1, $\nabla A^{\prime}=0$ holds.

Q.E. D.

Proposition 5.5. Suppose that $2 \leqq m \leqq 5, n \geqq m+13$, $\operatorname{Spec}(M, N)=\operatorname{Spec}\left(M^{\prime}, N\right)$ and that $\int_{M} K_{N} \geqq \int_{M} K_{N}^{\prime}$ holds. If $M$ has a constant curvature $k$ and its second fundamental tensor is parallel, then $M^{\prime}$ has also the constant curvature $k$, its second 
fundamental tensor parallel and $\int_{M} K_{N}=\int_{M^{\prime}} K_{N}^{\prime}$ holds.

Proof. From the assumption and Corollary 4.2

$$
\begin{aligned}
& C_{1}(m, n) \tau^{2}(M)-\frac{7}{12} K_{N}(M) \\
& =\frac{n-m}{180}\left(\left|C^{\prime}\right|^{2}+\frac{6-m}{m-2}\left|G^{\prime}\right|^{2}\right)\left(M^{\prime}\right)+C_{1}(m, n) \tau^{\prime 2}\left(M^{\prime}\right)+\frac{1}{12}\left(6 T^{\prime}-7 K_{N}^{\prime}\right)\left(M^{\prime}\right) \\
& \geqq C_{1}(m, n) \tau^{\prime 2}\left(M^{\prime}\right)-\frac{7}{12} K_{N}(M) .
\end{aligned}
$$

Thus $\tau^{2}(M) \geqq \tau^{12}\left(M^{\prime}\right)$. The rest of the proof proceeds in the same way as that of Proposition 5.4.

Proposition 5.6. Suppose that $2 \leqq m \leqq 5, n \geqq m+13, \operatorname{Spec}(M, N)=\operatorname{Spec}\left(M^{\prime}, N\right)$ and that $\int_{M} K_{N} \leqq \int_{M} K_{N}^{\prime}$ holds. If $M$ is isotropic and has a constant curvature $k$, then $M^{\prime}$ is also isotropic, has the constant curvature $k$, and $\int_{M} K_{N}=\int_{M} K_{N}^{\prime}$ holds.

Proof. From Lemma 1.2, $m L_{N}(M)=K_{N}(M) \leqq K_{N}^{\prime}\left(M^{\prime}\right) \leqq m L_{N}^{\prime}\left(M^{\prime}\right)$, thus $L_{N}(M)$ $\leqq L_{N}^{\prime}\left(M^{\prime}\right)$. Then the rest of the proof proceeds in the same way as that of Proposition 5.4.

Q.E.D.

Proposition 5.7. Suppose that $2 \leqq m \leqq 5, n \geqq m+13, \operatorname{Spec}(M, N)=\operatorname{Spec}\left(M^{\prime}, N\right)$ and that $\int_{M}\left(6 L_{N}-K_{N}\right) \leqq \int_{M},\left(6 L_{N}^{\prime}-K_{N}^{\prime}\right)$ holds. If $M$ has a constant curvature $k$, then so does $M^{\prime}$ and $\int_{M}\left(6 L_{N}-K_{N}\right)=\int_{M}\left(6 L_{N}^{\prime}-K_{N}^{\prime}\right)$ holds.

This Proposition can be easily proved as a consequence of the above arguments. Since from Lemma 1.2, $\int_{M}\left(6 L_{N}-K_{N}\right)=0$ holds for a 6 -dimensional isotropic submanifold Mof constant curvature, we have the following corollary.

COROLlary 5.8. In case $m=6$ and $n \geqq 19$, the following is a spectral property, The immersion is isotropic and the sectional curvature a constant $k$.

COROLlary 5.9. If a full minumal submanifold $M$ of $S^{19}\left[S^{26}\right]$ has the same spectrum with the 5-dimensional [6-dimensional] Veronese manifold $V^{5}\left[V^{6}\right]$, then $M$ is itself $V^{5}\left[V^{6}\right]$.

Proof. We shall prove Corollary 5.9 for 5-dimensional submanifolds, using Proposition 5.4. Suppose that $M$ is a full minimal submanifold of $S^{19}$ and isospectral with $V^{5} . \quad V^{5}$ is isotropic and has the constant curvature 5/12. Moreover its second 
fundamental tensor is parallel. Then $M$ has the same property (note that $19 \geqq 5+13$ ). Moreover, since $\operatorname{vol}(M)=\operatorname{vol}\left(V^{5}\right)$, the underlying manifold of $M$ is $S^{5}(2 \sqrt{3 / 5})$ and $M$ can be considered as $V^{5}$. Corollary 5.9 for 6-dimensional submanifolds can be $\begin{array}{ll}\text { proved in a similar way as a consequence of Corollary 5.8. } & \text { Q. E. D. }\end{array}$

Remark. We cannot apply Proposition 5.4 to characterizing $V^{3}$ or $V^{4}$ by reason of the restriction on the codimension (i. e. $n \geqq m+13$ ).

THEOREM 5.10. If a full minimal submanifold $M$ of $S^{8}$ has the same spectrum with the 3-dimensional Veronese manifold $V^{3}$ and $\int_{M} \tau^{2} \leqq 54 \sqrt{\frac{2}{3}} \pi^{2}=\int_{V^{3}} \tau^{\prime 2}$ holds, then $M$ is itself $V^{3}$.

Proof. Let $\tau^{\prime}, K_{N}^{\prime}$ and so on denote the scalar curvature, normal scalar curvature and so on of $V^{3} . \quad V^{3}$ is isotropic and has the constant curvature $3 / 8$. Moreover its second fundamental tensor is parallel. Then

Therefore

$$
\tau^{2}(M) \leqq \tau^{\prime 2}\left(V^{3}\right) \text {, thus } \tau^{2}(M)=\tau^{\prime 2}\left(V^{3}\right) \text {, and } L_{N}(M) \geqq L_{N}^{\prime}\left(V^{3}\right) \text {. }
$$

$$
\begin{aligned}
90 L_{N}^{\prime}\left(V^{3}\right) & =10\left(|C|^{2}+3|G|^{2}\right)(M)+30\left(6 L_{N}-K_{N}\right)(M) \\
& \geqq 90 L_{N}(M) \geqq 90 L_{N}^{\prime}\left(V^{3}\right) .
\end{aligned}
$$

Therefore we have the following conclusions; $L_{N}(M)=L_{N}^{\prime}\left(V^{3}\right), \quad C=0, \quad G=0$, $\left(3 L_{N}-K_{N}\right)(M)=0 ; M$ is isotropic and has the constant curvature $3 / 8$; its second fundamental tensor is parallel. Since $\operatorname{vol}(M)=\operatorname{vol}\left(S^{3}(2 \sqrt{ } 2 / 3)\right), M=V^{3}$. Q. E. D.

The following Theorem can be proved in a similar way.

THEOREM 5.11. If a full minimal submanifold $M$ of $S^{\prime 3}$ has the same spectrum with the 4-dimensional Veronese manifold $V^{4}$ and $\int_{M} \tau^{2} \leqq 384 \pi^{2}=\int_{V^{4}} \tau^{\prime 2}$ holds, then $M$ is itself $V^{4}$.

Proposition 5.12. Suppose $\operatorname{Spec}(M, N)=\operatorname{Spec}\left(M^{\prime}, N\right)$ and $m \geqq 7$. Assume that $M$ is Einstein, $M^{\prime}$ is conformally flat and isotropic and that $\int_{M} K_{N} \leqq \int_{M^{\prime}} K_{N}^{\prime}$ holds. Then $M$ and $M^{\prime}$ have the same constant curvature and $M$ is isotropic. Moreover if the second fundamental tensor of $M$ is parallel, so is that of $M^{\prime}$. tion,

Proof. Since an isotropic minimal submanifold is Einstein, from the assump-

$$
\begin{aligned}
\frac{6-m}{12 m} K_{N}^{\prime}\left(M^{\prime}\right) & =\frac{n-m}{180}|C|^{2}(M)+\frac{1}{12}\left(6 L_{N}-K_{N}\right)(M) \\
& \geqq \frac{6-m}{12 m} K_{N}(M) \geqq \frac{6-m}{12 m} K_{N}^{\prime}\left(M^{\prime}\right) .
\end{aligned}
$$


Thus $K_{N}(M)=K_{N}^{\prime}\left(M^{\prime}\right)$, and $M$ is conformally flat and isotropic. Moreover if $\nabla A=0$, then from Lemma $1.2, m c S(M)=(m+1) L_{N}(M)$. Since $m L_{N}(M)=K_{N}(M)$ $=K_{N}^{\prime}\left(M^{\prime}\right)=m L_{N}^{\prime}\left(M^{\prime}\right), m c S^{\prime}\left(M^{\prime}\right)=(m+1) L_{N}^{\prime}\left(M^{\prime}\right)$ holds, thus $\nabla A^{\prime}=0$.

Q.E.D.

The following Propositions 13, 14 and 15 can be proved in a similar way, so we shall omit their proofs.

Proposition 5.13. Suppose $\operatorname{Spec}(M, N)=\operatorname{Spec}\left(M^{\prime}, N\right)$. If $M$ has a constant curvature $k$, and $M^{\prime}$ is Einstern (resp. conformally flat, $m \geqq 7$ and $n \leqq m+12$ ) and if $\int_{M}\left(6 L_{N}-K_{N}\right) \leqq \int_{M}\left(6 L_{N}^{\prime}-K_{N}^{\prime}\right)\left(\operatorname{resp} . \int_{M}\left(6 L_{N}-K_{N}\right) \geqq \int_{M}\left(6 L_{N}^{\prime}-K_{N}^{\prime}\right)\right)$ holds, then $M^{\prime}$ has the same constant curvature $k$ and $\int_{M}\left(6 L_{N}-K_{N}\right)=\int_{M^{\prime}}\left(6 L_{N}^{\prime}-K_{N}^{\prime}\right)$ holds.

Proposition 5.14 Suppose $\operatorname{Spec}(M, N)=\operatorname{Spec}\left(M^{\prime}, N\right)$ and $m \geqq 7$. If $M$ isotropuc, has a constant curvature $k$, and if $M^{\prime}$ is Einstern and $\int_{M} K \geqq \int_{M} K_{N}^{\prime}$ holds, then $M^{\prime}$ is also isotropic and has the constant curvature $k$. Moreover if the second fundamental tensor of $M$ is parallel, so is that of $M^{\prime}$.

Proposition 5.15. Suppose $\operatorname{Spec}(M, N)=\operatorname{Spec}\left(M^{\prime}, N\right)$. If $M$ has a constant curvature $k$ and a parallel second fundamental tensor, and if $M^{\prime}$ is Einstern and $\int_{M} K_{N} \geqq \int_{M^{\prime}} K_{N}^{\prime}$ holds, then $M^{\prime}$ has also the constant curvature $k$ and a parallel second fundmental tensor.

From Propositions 5.13 and 5.15, we obtain

COROLlary 5.16. If a full Einstein minmmal submanifold $M$ of $S^{m+\frac{m(m-1)}{2}-1}$ has the same spectrum with the m-dimensional Veronese manifold $V^{m}$, and

$$
\int_{M}\left(6 L_{N}-K_{N}\right) \geqq \frac{(m-1)(m+2) m^{2}(6-m)}{2(m+1)^{2}}\left(\frac{2(m+1)}{m}\right)^{m / 2} \omega_{m}=\int_{V m}\left(6 L_{N}^{\prime}-K_{N}^{\prime}\right)
$$

or

$$
\int_{M} K_{N} \leqq \frac{m^{3}(m-1)(m+2)}{2(m+1)^{2}}\left(\frac{2(m+1)}{m}\right)^{m / 2} \omega_{m}=\int_{V m} K_{N}^{\prime}
$$

holds, then $M$ is itself $V^{m}$. In particular if a full isotropic minımal submantold $M$ of $S^{m+\frac{m(m+1)}{2}-1}$ has the same spectrum with $V^{m}$, then $M$ is itself $V^{m}(m \geqq 3)$.

The latter statement follows from the observation that since an isotropic minimal submanifold is Einstein and $K_{N}=\frac{2}{m+2} S^{2}+L_{N}$ holds ([15]), $\begin{gathered}n-m \\ 180\end{gathered}$ $\int_{M}|C|^{2}+\frac{5}{12} \int_{M} K_{N}$ is its spectral invariant. Thus let $K_{N}^{\prime}$ be the normal scalar curvature of $V^{m}$, then 


$$
\frac{n-m}{180} \int_{M}|C|^{2}+\frac{5}{12} \int_{M} K_{N}=\frac{5}{12} \int_{V^{m}} K_{N}^{\prime} \geqq \frac{5}{12} \int_{M} K_{N} .
$$

Thus the assumption on the normal scalar curvature is automatically satisfied.

Now we state some propositions which are related with Einstein property.

Proposition 5.17. Suppose that $n \geqq 18, M$ and $M^{\prime}$ be 4-dimensional orlented minimal submanifolds of $N$, and that $\operatorname{Spec}(M, N)=\operatorname{Spec}\left(M^{\prime}, N\right)$ holds. If $M$ is Einstein and its second fundamental tensor parallel,

$$
\begin{aligned}
& \frac{8(n-4)}{15} \pi^{2} \cdot \chi(M)-\frac{7}{4} \int_{M} K_{N} \geqq \frac{8(n-4)}{15} \pi^{2} \cdot \chi\left(M^{\prime}\right)-\frac{7}{4} \int_{M^{\prime}} K_{N}^{\prime}, \\
& \frac{4(n-4)}{5} \pi^{2} \cdot \operatorname{sign}(M)+\frac{7}{12} \int_{M} K_{N} \leqq \frac{4(n-4)}{15} \pi^{2} \cdot \operatorname{sign}\left(M^{\prime}\right)+\frac{7}{12} \int_{M} K_{N}^{\prime}
\end{aligned}
$$

hold, and in either inequality the equality is attained if and only if $M^{\prime}$ is Einstein and its second fundamental tensor is parallel.

Proof. We prove the first inequality. The second inequality can be proved in a similar way. From the assumption and using a spectral invariant given in the proof of Proposition 4.3,

$$
\begin{aligned}
& \frac{8(n-4)}{45} \pi^{2} \cdot \chi(M)+\left(C_{1}(4, n)-\frac{n-4}{1080}\right) \tau^{2}(M)-\frac{7}{12} K_{N}(M) \\
& =\frac{8(n-4)}{45} \pi^{2} \cdot \chi\left(M^{\prime}\right)+\left(C_{1}(4, n)-\frac{n-4}{1080}\right) \tau^{\prime 2}\left(M^{\prime}\right)+\frac{n-4}{60}\left|G^{\prime}\right|^{2}\left(M^{\prime}\right) \\
& \quad+\frac{1}{12}\left(6 T^{\prime}-7 K_{N}^{\prime}\right)\left(M^{\prime}\right) \\
& \geqq \frac{8(n-4)}{45}-\pi^{2} \cdot \chi\left(M^{\prime}\right)+\left(C_{1}(4, n)-\frac{n-4}{1080}\right) \tau^{2}(M)-\frac{7}{12} K_{N}^{\prime}\left(M^{\prime}\right),
\end{aligned}
$$

where we note that $C_{1}(4, n)>\frac{n-4}{1080}$ follows from $n \geqq 18$, and $\tau^{\prime 2}\left(M^{\prime}\right) \geqq \tau^{2}(M)$ holds because $\tau$ is constant and $\tau(M)=\tau^{\prime}\left(M^{\prime}\right)$ Q.E. D.

Proposition 5.18. Suppose that $n \geqq 14, M$ and $M^{\prime}$ be 4-dimensional oriented minimal submanifold of $N$ and that $\operatorname{Spec}(M, N)=\operatorname{Spec}\left(M^{\prime}, N\right)$ holds. If $\tau$ is constant and $\int_{M}\left(6 L_{N}-K_{N}\right) \leqq \int_{M}\left(6 L_{N}^{\prime}-K_{N}^{\prime}\right)$ holds,

$$
\operatorname{sign}(M)+\frac{2}{9} \chi(M) \leqq \operatorname{sign}\left(M^{\prime}\right)+\frac{2}{9} \chi\left(M^{\prime}\right)
$$

holds and the equality is attanned if and only if $\tau=\tau^{\prime}$ and $\int_{M}\left(6 L_{N}-K_{N}\right)=$ 
$\int_{M^{\prime}}\left(6 L_{N}^{\prime}-K_{N}^{\prime}\right)$ hold. If the second fundamental tensor of $M$ is parallel and $\int_{M} K_{N} \geqq \int_{M^{\prime}} K_{N}^{\prime}$ holds, the same inequality is derved and in that case the equality is attained if and only if the second fundamental tensor of $M^{\prime}$ is parallel and $\int_{M} K_{N}=\int_{M^{\prime}} K_{N}^{\prime}$ holds.

Proof. From Proposition 4.3

$$
\begin{aligned}
& \left(C_{1}(4, n)+\frac{7(n-4)}{2160}\right)\left(\tau^{2}(M)-\tau^{\prime 2}\left(M^{\prime}\right)\right)+\frac{1}{12}\left(6 L_{N}-K_{N}\right)(M)-\frac{1}{12}\left(6 L_{N}^{\prime}-K_{N}^{\prime}\right)\left(M^{\prime}\right) \\
& =\frac{2(n-4)}{5} \pi^{2} \cdot\left(\operatorname{sign}(M)-\operatorname{sign}\left(M^{\prime}\right)\right)+\frac{4(n-4)}{45} \pi^{2} \cdot\left(\chi(M)-\chi\left(M^{\prime}\right)\right) .
\end{aligned}
$$

And from the assumption, $C_{1}(4, n)+\frac{7(n-4)}{2160} \geqq 0$. Thus the first statement follows immediately. The second statement can be proved in the same way.

Q.E.D.

Proposition 5.19. Suppose that $n \geqq 18, \operatorname{dim} M=4$ and $\operatorname{Spec}(M, N)=\operatorname{Spec}\left(M^{\prime}, N\right)$ holds. If $M$ is Einstern,

$$
\frac{8(n-4)}{15} \pi^{2} \cdot \chi(M)+\frac{1}{4} \int_{M}\left(6 L_{N}-K_{N}\right) \geqq \frac{8(n-4)}{15} \pi^{2} \cdot \chi\left(M^{\prime}\right)+\frac{1}{4} \int_{M}\left(6 L_{N}^{\prime}-K_{N}^{\prime}\right)
$$

holds and the equality is attarned if and only if $M^{\prime}$ is Einstern.

Finally we state some miscellaneous properties.

The following Proposition was obtained by H. Donnelly ([8]).

Proposition 5.20 If $\operatorname{Spec}(M, N)=\operatorname{Spec}\left(M^{\prime}, N\right)$ holds and $M$ is totally geodesic, then $M^{\prime}$ is also totally geodesic.

This follows from the fact that $\int_{M} S$ is a spectral invariant and it becomes 0 when and only when $M$ is totally geodesic.

The following two Propositions can be easily proved, so we shall omit their proofs.

Proposition 5.21. Suppose that $m=6, n \geqq 19, \operatorname{Spec}(M, N)=\operatorname{Spec}\left(M^{\prime}, N\right)$ and $\int_{M} K_{N} \geqq \int_{M} K_{N}^{\prime}$ hold. If $M$ is conformally flat and its second fundamental tensor parallel, then $M^{\prime}$ is also conformally flat, its second fundamental tensor parallel and $\tau=\tau^{\prime}$ holds.

Proposition 5.22. Suppose $m=6$ and $\operatorname{Spec}(M, N)=\operatorname{Spec}\left(M^{\prime}, N\right)$. Assume that 
$M$ is conformally flat, and that $\tau$ is constant and $n \geqq 19$, or that $\tau^{\prime}$ is constant and $n \leqq 18$, then $\int_{M}\left(6 L_{N}-K_{N}\right) \geqq \int_{M^{\prime}}\left(6 L_{N}^{\prime}-K_{N}^{\prime}\right)$ holds and the equality is attained if and only if $M^{\prime}$ is conformally flat and $\tau=\tau^{\prime}$ holds.

THEOREM 5.23. Suppose that an m-dimensional minımal submanifold $M$ of $S^{2 m-1}$ has the same spectrum with $M_{1, \ldots, 1}$, and $\int_{M} 6 T \geqq 7 \int_{M} K_{N}$ holds. If $M$ is Einstein, or if its scalar curvature is constant and $m<6$, then $M$ is itself $M_{1, \ldots, 1}$.

Proof. Under the assumption

$$
\begin{aligned}
0 & =\frac{n-m}{180}\left(|C|^{2}+\frac{6-m}{m-2}|G|^{2}\right)(M)+\frac{1}{12}\left(6 T-7 K_{N}\right)(M) \\
& \geqq \frac{1}{12}\left(6 T-7 K_{N}\right)(M) \geqq 0 .
\end{aligned}
$$

Thus $M$ is flat, and by a result of S. T. Yau ([26]) a compact $m$-dimensional fiat minimal submanifold of $S^{2 m-1}$ is just $M_{1, \ldots, 1}$.

Q.E.D.

THEOREM 5.24. If a minimal submannfold $M$ of $S^{5}$ has the same spectrum with the Clifford hypersurface $M_{2,2}$ and its Euler number $\chi(M) \leqq 4=\chi\left(M_{2,2}\right)$, then $M$ is itself $M_{2,2}$.

Proof. For a minimal hypersurface, $L_{N}=S^{2}$ and $K_{N}=0$ hold. Thus, using an invariant given in the proof of Proposition 4.3, for a 4-dimensional minimal hypersurface $M$ of $S^{5}$

$$
32 \pi^{2} \cdot \chi(M)+3|G|^{2}(M)+\frac{249}{4} \tau^{2}(M)
$$

is a spectral invariant. Thus if $\operatorname{Spec}\left(M, S^{5}\right)=\operatorname{Spec}\left(M_{2,2}, S^{5}\right)$ and $\chi(M) \leqq 4$, then $M$ is also Einstein and $S=m$. Therefore $M=M_{2,2}$ Q. E. D.

THEOREM 5.25. Suppose that $2 \leqq m_{1}^{\prime} \leqq m_{2}^{\prime} \leqq \cdots \leqq m_{k}^{\prime}, \quad m=m_{1}^{\prime}+\cdots+m_{k}^{\prime}$, and Spec $\left(M_{m_{1}^{\prime}, \cdots, m_{k}^{\prime}}, S^{m+k-1}\right)=\operatorname{Spec}\left(M, S^{m+k-1}\right)$ holds. If $M$ has a nonnegatvve curvature and $\int_{M} \tau^{2} \leqq \int_{M_{m_{1}^{\prime}, \cdot m_{k}^{\prime}}} \tau^{\prime 2}$ holds, then $M$ is $M_{m_{1}^{\prime}, \cdots, m_{k}^{\prime}}$.

Proof. Since $M_{m_{1}^{\prime}, \cdots, m_{k}^{\prime}}$ has a constant scalar curvature, first we can assert that $\tau=\tau^{\prime}$ i. e. $S=S^{\prime}=(k-1) m$ holds. Let $K$ be a function on $M$ which assigns to each point the minimum value of sectional curvatures at that point. From the assumption, $K \geqq 0$. Thus $S \geqq(k-1) m(1-2 K)$. Then by a theorem of Yau ([26]), the second fundamental tensor of $M$ is parallel, and $S=(k-1) m(1-2 K)$ holds because $M$ is not totally geodesic. Thus $K \equiv 0$, and again by a result of Yau ([26]), $M$ is a product of spheres ; $M=M_{m_{1}, \cdots, m_{k}}, 1 \leqq m_{1} \leqq \cdots \leqq m_{k}, m=m_{1}+\cdots+m_{k}$. 
The normal bundle of $M_{m_{1}, \ldots, m_{k}}$ is globally parallelizable, and its $\tilde{A}$ is a scalar transformation. Thus, from Proposition 2.7, $\operatorname{Spec}\left(M_{m_{1}, \ldots, m_{k}}, S^{m+k-1}\right)=$ $\operatorname{Spec}\left(M_{m_{1}^{\prime}, \cdots, m_{k}^{\prime}}, S^{m+k-1}\right)$ is equivalent to $\operatorname{Spec}\left(M_{m_{1}, \ldots, m_{k}}\right)=\operatorname{Spec}\left(M_{m_{1}^{\prime}, \cdots, n_{k}^{\prime}}\right)$, where $\operatorname{Spec}(M)$ denotes the complete set of eigenvalues of $-\nabla^{2}$ acting on functions on $M$. Spec $\left(M_{m_{1}^{\prime}, \cdots, m_{k}^{\prime}}\right)$ can be easily computed. Its eigenvalues between $2 m$ and $3 m$ are $2 m<2\left(1+1 / m_{k}^{\prime}\right) m \leqq \cdots \leqq 2\left(1+1 / m_{1}^{\prime}\right) m \leqq 3 m$, and the multiplicity of $2\left(1+1 / m_{\imath}^{\prime}\right) m$ is equal to $m_{\imath}^{\prime}\left(m_{\imath}^{\prime}+3\right) / 2$. Thus from $\operatorname{Spec}\left(M_{m_{1}, \ldots, m_{k}}\right)=\operatorname{Spec}\left(M_{m_{1}^{\prime}, \cdots, m_{k}^{\prime}}\right)$ we can conclude that $m_{1}=m_{1}^{\prime}, \cdots, m_{k}=m_{k}^{\prime}$.

Q.E.D.

\section{$\S 6$. Kaehlerian case.}

In this section, using the Kaehlerian spectral invariants given in $\S 4$, we obtain some spectral properties, and then characterize some concrete Kaehler submanifolds of a complex projective space.

Let $n$ be the complex dimension of the complex space form $N=N(c)$, and $m$ be the complex dimension of its compact Kaehler submanifold $M$.

First we note that in some situations those properties which appear general reduce to special ones, for example, Kaehler submanifolds with parallel second fundamental tensor or with constant holomorphic curvature in a complex space form of nonpositive holomorphic curvature are necessarily totally geodesic. And if $n-m<m(m+1) / 2$, a Kaehler submanifold of constant holomorphic curvature is also totally geodesic.

Proposition 6.1. Suppose that $m \leqq 6$ and $n \geqq m+7$, or that $m=7$ and $35 \leqq n$ $\leqq 51$, and that $\operatorname{Spec}(M, N)=\operatorname{Spec}\left(M^{\prime}, N\right)$ holds. Then if $M$ has a constant holomorphic curvature $\tilde{c}$, so does $M^{\prime}$.

Proof. Under the assumption $(n-m)(6-m)-15 m+150>0$ and $C_{4}(m, n)>0$. Thus, from (3) of Corollary 4.4

$$
\begin{aligned}
& C_{4}(m, n) \tau^{2}(M) \\
& =\frac{2(n-m)+45}{180}\left|B^{\prime}\right|^{2}\left(M^{\prime}\right)+\frac{(n-m)(6-m)-15 m+150}{90(m+2)}\left|G^{\prime}\right|^{2}\left(M^{\prime}\right)+C_{4}(m, n) \tau^{\prime 2}\left(M^{\prime}\right) \\
& \geqq C_{4}(m, n) \tau^{\prime 2}\left(M^{\prime}\right) .
\end{aligned}
$$

From $\tau(M)=\tau^{\prime}\left(M^{\prime}\right)$ and $\tau$ being constant, $\tau^{2}(M) \leqq \tau^{\prime 2}\left(M^{\prime}\right)$ holds, thus $\tau^{2}(M)=\tau^{\prime 2}\left(M^{\prime}\right)$, and $\tau=\tau^{\prime}, B^{\prime}=0, G^{\prime}=0$ hold.

Q.E. D.

Corollary 6.2. Complex Veronese manufolds $C V^{m}$ of dimensions from 3 to 7 are characterized by their spectra.

The case of $4 \leqq m \leqq 7$ is an immediate consequence of the above Proposition. The case of $m=3$ follows from the fact $C_{4}(3,9)>0$ even though $3+7>9$. Note that the complex codimension of $C V^{2}$ is just 3, which is the case out of our 
consideration.

Proposition 6.3. Suppose that $m \leqq 6$, or that $m=7$ and $35 \leqq n \leqq 51$, and that $\operatorname{Spec}(M, N)=\operatorname{Spec}\left(M^{\prime}, N\right)$ holds. Then if $M$ has a constant holomorphic curvature $\tilde{c}$ and $\int_{M} \tau^{2} \geqq \int_{M} \tau^{\prime 2}$ holds, $M^{\prime}$ has also the constant holomorphic curvature $\tilde{c}$.

From (3) of Corollary 4.4, we have

Proposition 6.4. Suppose that $9 \leqq m, n \geqq m+7$, and $\operatorname{Spec}(M, N)=\operatorname{Spec}\left(M^{\prime}, N\right)$ holds. If $M$ is Einstem, the Bochner curvature tensor of $M^{\prime}$ vanushes and $\int_{M} \tau^{2} \geqq \int_{M} \tau^{\prime 2}$ holds, then $M$ and $M^{\prime}$ have the same constant holomorphic curvature.

This follows from the fact that under the assumption,

$$
(n-m)(6-m)-15 m+150<0 \text { and } C_{4}(m, n)>0 .
$$

Corollary 6.5. If an Einstem Kaehler submannfold $M$ of $C P^{m+m(m+1) / 2}$ has the same spectrum with the m-dimensional complex Veronese manifold $\mathrm{CV}^{m}$ and $\int_{M} \tau^{2} \geqq m(m+1)^{2} \frac{8^{m} \pi^{m}}{4(m-1) !}=\int_{C V m} \tau^{\prime 2}$ holds, then $M$ is utself $C V^{m}(m \geqq 9)$.

Proposition 6.6. Suppose $\operatorname{Spec}(M, N)=\operatorname{Spec}\left(M^{\prime}, N\right)$. If $M$ has a constant holomorphac curvature $\tilde{c}$, then $\int_{M} T \leqq \int_{M} T^{\prime}$ holds and the equality is attamed if and only if $M^{\prime}$ has the constant holomorphic curvature $\tilde{c}(m \geqq 2)$.

Proof. From (4) of Corollary 4.5 and the assumption

$$
\begin{aligned}
& \frac{m+2}{m(m+1)} \tau^{2}(M)-T(M) \\
& =\frac{1}{2}\left|B^{\prime}\right|^{2}\left(M^{\prime}\right)+\frac{2 m+8}{m+2}\left|G^{\prime}\right|^{2}\left(M^{\prime}\right)+\frac{m+2}{m(m+1)} \tau^{\prime 2}\left(M^{\prime}\right)-T^{\prime}\left(M^{\prime}\right) \\
& \geqq \frac{m+2}{m(m+1)} \tau^{\prime 2}(M)-T^{\prime}\left(M^{\prime}\right) \geqq \frac{m+2}{m(m+1)} \tau^{2}(M)-T^{\prime}\left(M^{\prime}\right) .
\end{aligned}
$$

Thus we obtain the Proposition.

Q.E.D.

COROLlARY 6.7. If a Kaehler submanfold $M$ of $C P^{m+m(m+1) / 2}$ has the same spectrum with the m-dimensional complex Veronese mannfold $C V^{m}$ and the second fundamental tensor of $M$ is parallel, then $M$ is itself $C V^{m}(m \geqq 3)$.

From (5) of Corollary 4.5 we have

Proposition 6.8. Suppose that $n \leqq m+6+27 / m-1$ and $\operatorname{Spec}(M, N)=$ 
$\operatorname{Spec}\left(M^{\prime}, N\right)$ holds. If $M$ is Einstern, then $\int_{M} T \leqq \int_{M^{\prime}} T^{\prime}$ holds and the equality is attained if and only if $M^{\prime}$ is Einstern. In particular if $M$ is Einstern and the second fundamental tensor of $M^{\prime}$ is parallel, then $M^{\prime}$ is also Einstern and the second fundamental tensor of $M$ is parallel.

Proposition 6.9. Suppose $\operatorname{Spec}(M, N)=\operatorname{Spec}\left(M^{\prime}, N\right)$. If $M$ is Einstern and $\int_{M} \tau^{2} \geqq \int_{M} \tau^{\prime 2}$ holds, then $\int_{M} T \leqq \int_{M} T^{\prime}$ holds and the equality is attained if and only if $M^{\prime}$ is Einstein.

Proposition 6.10. If $\operatorname{Spec}(M, N)=\operatorname{Spec}\left(M^{\prime}, N\right)$ holds and $M$ is totally geodesic, then $M^{\prime}$ is also totally geodesic.

From Corollary 4.5 we have

Proposition 6.11. If $M$ is a complex curve in $N$, then the following are its spectral invariants;

$$
\int_{M} \tau^{2}, \int_{M} T
$$

COROLlary 6.12. For complex curves in $N$, the Gaussian curvature being a constant $K$ is a spectral property.

COROLlary 6.13. If a full Kaehler submanfold $M$ of $C P^{n}$ has the same spectrum with $C V_{n-1}^{1}$, then $M$ is $C V_{n-1}^{1}$.

Proposition 6.14. Suppose that $m=2, n \geqq 5$ (resp. $n \geqq 11)$, and $\operatorname{Spec}(M, N)=$ $\operatorname{Spec}\left(M^{\prime}, N\right)$ holds. If $M$ is Einstern, then $\operatorname{sign}(M) \leqq \operatorname{sign}\left(M^{\prime}\right)\left(\right.$ resp. $\left.\chi(M) \geqq \chi\left(M^{\prime}\right)\right)$ holds and the equality is attained if and only if $M^{\prime}$ is Einstein.

Proof. In terms of $B, G$ and $\tau, \chi(M)$ and $\operatorname{sign}(M)$ are expressed as

$$
\begin{aligned}
& \chi(M)=\frac{1}{32 \pi^{2}}\left(|B|^{2}-2|G|^{2}+\frac{1}{3} \tau^{2}\right)(M), \\
& \operatorname{sign}(M)=\frac{-1}{48 \pi^{2}}\left(|B|^{2}-\frac{1}{6} \tau^{2}\right)(M) .
\end{aligned}
$$

From (2) of Corollary 4.5 and these expressions we obtain the spectral invariants ;

$$
\begin{aligned}
& -12(8 n+164) \pi^{2} \cdot \operatorname{sign}(M)+2(n+28)|G|^{2}(M)+\frac{33 n-156}{6} \tau^{2}(M), \\
& 16(2 n+41) \tau^{2} \cdot \chi(M)+3(n+23)|G|^{2}(M)+\frac{9 n-93}{4} \tau^{2}(M) .
\end{aligned}
$$


If $n \geqq 5$ (resp. $n \geqq 11$ ) and $M$ is Einstein, the coefficients of $\tau^{2}$ in the upper (resp. lower) invariant is positive and $\tau^{2}(M) \leqq \tau^{\prime 2}\left(M^{\prime}\right)$ holds. Thus we obtain the Proposition.

Q.E.D.

Proposition 6.15. Suppose that $m=2$ and $\operatorname{Spec}(M, N)=\operatorname{Spec}\left(M^{\prime}, N\right)$ holds. If the scalar curvature of $M$ is constant and the second fundamental tensor of $M^{\prime}$ is parallel, then $2 \operatorname{sign}\left(M^{\prime}\right)+\chi\left(M^{\prime}\right) \geqq 2 \operatorname{sign}(M)+\chi(M)$ holds and the equality is attained if and only if the second fundamental tensor of $M$ is parallel.

Proof. From (4) of Corollary 4.5 and the expressions for $\chi$ and sign given in the proof of Proposition 6.14,

$$
\begin{aligned}
& -24 \pi^{2} \cdot \operatorname{sign}(M)+3|G|^{2}(M)+\frac{3}{4} \tau^{2}(M)-T(M), \\
& 16 \pi^{2} \cdot \chi(M)+4|G|^{2}(M)+\frac{1}{2} \tau^{2}(M)-T(M)
\end{aligned}
$$

are the spectral invariants. From these, the following is also spectral invariant;

$$
96 \pi^{2} \cdot \operatorname{sign}(M)+48 \pi^{2} \cdot \chi(M)-\frac{3}{2} \tau^{2}(M)+T(M) .
$$

Using this we obtain the Proposition.

Q.E. D.

THEOREM 6.16. If a Kaehler submanfold $M$ of $C P^{3}$ has the same spectrum with the 2-dimensional quadratic $Q^{2}$ and $31 \chi(M)+117 \operatorname{sign}(M) \leqq 124=31 \chi\left(Q^{2}\right)+$ $117 \operatorname{sign}\left(Q^{2}\right)$ holds, then $M$ is itself $Q^{2}$.

Proof. Using the invariants given in the proof of Proposition 6.15, we obtain a new invariant;

$$
16 \pi^{2} \cdot \chi(M)+24 \pi^{2} \cdot \operatorname{sign}(M)+|G|^{2}(M)-\frac{1}{4} \tau^{2}(M) .
$$

This, together with the invariant given in the proof of Proposition 6.14 (in case $n=3$ ), gives a new invariant;

$$
31 \chi(M)+117 \operatorname{sign}(M)-\frac{3}{16 \pi^{2}} \tau^{2} .
$$

Using this we obtain the Theorem.

Q.E. D.

\section{REFERENCES}

[1] M. Berger, P. Gauduchon et E. Mazet, Le Spectre d'une Variété riemannienne, Lect. Notes in Math. Springer-Verlag, 194.

[2] D.D. BleEcker and J.L. Weiner, Extrinsic Bounds on $\lambda_{1}$ of $\Delta$ on a Compact Manifold, Comment. Math. Helv. 39 (51), 601-609.

[3] E. CALABI, Minimal Immersions of Surfaces in Euclidean Spheres, J. Diff. 
Geometry 1 (1967), 111-125.

[4] B. Cilen, Geometry of Submanifolds, Marcel Dekker, New York, 1973.

[5] S.S. Chern, M. DoCARmo ANd S. Kobayasif, Minimal submanifolds of a sphere with second fundamental form of constanc length, Functional Analysis and Related Fields, Springer, Berlin, 1970, 59-75.

[6:- M. P. DoCARmo ANd N. R. WALlaCi, Minımal immersions of spheres into spheres, Ann. of Math. 95 (1971), 43-62.

[7] H. Donnelly, Topology and Einstein Kaehler Metrics, J. Diff. Geometry, 11 (1976), 259-264.

[8] H. Donvelly, Spectral Invariants of The Second Variation Operator, Illinois Journ. of Math., vol. 21 (1977), 185-189.

[9] P.B. Gilkey, The Spectral Geometry of a Riemannıan Manıfold, J. Diff. Geometry, 10 (1975), 601-608.

[10- P. B. Gilkey, The Spectral Geometry of Symmetrıc Spaces, Trans. Amer. Math. Soc., vol. 225 (1977), 341-353.

[11] T. Hasegawa, The Equation of a Vibrating General Membrane, to appear.

[12] T. Iron, On Minımal Surfaces in a Riemannian Manifold of Constant Curvature, Koda1 Math. Sem. Rep. 25 (1973), 202-214.

[13] 'Г. Iтоі, On Veronese manifolds, J. Math. Soc. Japan, vol. 27 (1975), 497-506.

[14] T. I'or ANd K. Ogiue, Isotropic Immersions, J. Diff. Geometry, 8 (1973), 305316.

[15 T. ITOII AND K. OGiue, Isotropic Immersions and Veronese Manifolds, Trans. Amer. Math. Soc., vol. 209 (1975), 109-117.

[16] M. KAc, Can one hear the shape of a drum? Amer. Math. Monthly, 73 (April, 1966), 1-23.

[17 Y. KimurA, The nullity of compact Kähler submanifolds in a complex projective space, J. Math. Soc. Japan, vol. 29, 561-580.

[18, M. Kos, Complex submanifolds with constant scalar curvature in a Kaehler manifold, J. Math. Soc. Japan, vol. 27, 76-81.

[19] H.B. LAwson, Local rigidity theorems for mintmal hypersurfaces, Ann. of Math., 89, 187-197 (1969).

[20] H.F. MCKeAn ANd I. M. Singer, Curvature and the eigenvalues of the Laplacran, J. Diff. Geometry, 1 (1967), 43-69.

[21] K. OGILe, Differential Geometry of Kaehler Submanifolds, Advances in Math., 13, 73-114 (1974).

[22] T. Orstki, Minimal hypersurfaces in a Riemannian manifold of constant curvature, Amer. J. Math., 92 (1970), 145-173.

[23] K. Sakamoto, Planar Geodesic Immersions, Tôhoku Math. J., 29 (1977), 25-56.

[24] J. Simons, Minimal varıetıes in Riemannian manıfolds, Ann. of Math. 88 (1968), $62-105$.

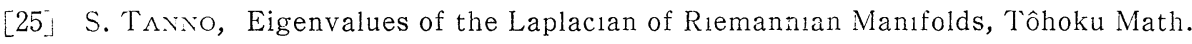
J., 25 (1973), 391-403.

[26. S. T. YAU, Submanifolds with Constant Mean Curvature Il, Amer. J. Math., vol. $97,76-100$.

Department of Mathematics

Tokyo Institute of Technology. 